(NASA-CR-194416) STORMTIME

TRANSPURT OF RING CURRENT ANO RAOIATION BELT IONS (AEROSPACE Corp.) $19 \mathrm{p}$

$$
\begin{aligned}
& \begin{array}{l|l}
\text { N94-13793 } & \begin{array}{l}
\text { AEROSPACE REPORT NO } \\
\text { ATR-92(7190)-2 }
\end{array}
\end{array} \\
& N-4 B-3 \\
& 186097
\end{aligned}
$$

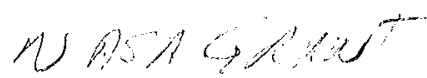

\title{
Stormtime Transport of Ring Current and Radiation Belt Ionc
}

\author{
NASA-CR-194416 \\ 19940009320
}

Prepared by

M. W. CHEN, M. SCHULZ, L. R. LYONS, and D. J. GORNEY

Space and Environment Technology Center

Technology Operations

15 April 1993

Prepared for

NASA HEADQUARTERS

Washington, DC 20546

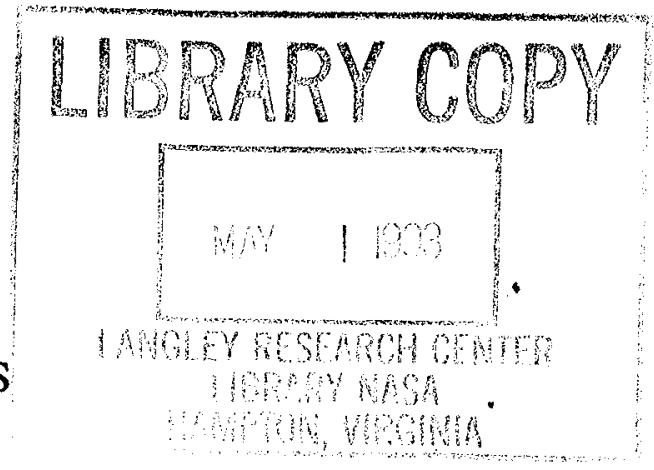

Grant No. NAGW 2126

Engineering and Technology Group

THE AEROSPACE CORPORATION

El Segundo, California

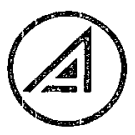




\section{TECHNOLOGY OPERATIONS}

The Aerospace Corporation functions as an "architect-engineer" for national security programs, specializing in advanced military space systems. The Corporation's Technology Operations supports the effective and timely development and operation of national security systems through scientific research and the application of advanced technology. Vital to the success of the Corporation is the technical staff's wide-ranging expertise and its ability to stay abreast of new technological developments and program support issues associated with rapidly evolving space systems. Contributing capabilities are provided by these individual Technology Centers:

Electronics Technology Center: Microelectronics, solid-state device physics, VLSI reliability, compound semiconductors, radiation hardening, data storage technologies, infrared detector devices and testing; electro-optics, quantum electronics, solid-state lasers, optical propagation and communications; $\mathrm{cw}$ and pulsed chemical laser development, optical resonators, beam control, atmospheric propagation, and laser effects and countermeasures; atomic frequency standards, applied laser spectroscopy, laser chemistry, laser optoelectronics, phase conjugation and coherent imaging, solar cell physics, battery electrochemistry, battery testing and evaluation.

Mechanics and Materials Technology Center: Evaluation and characterization of new materials: metals, alloys, ceramics, polymers and their composites, and new forms of carbon; development and analysis of thin films and deposition techniques; nondestructive evaluation, component failure analysis and reliability; fracture mechanics and stress corrosion; development and evaluation of hardened components; analysis and evaluation of materials at cryogenic and elevated temperatures; launch vehicle and reentry fluid mechanics, heat transfer and flight dynamics; chemical and electric propulsion; spacecraft structural mechanics, spacecraft survivability and vulnerability assessment; contamination, thermal and structural control; high temperature thermomechanics, gas kinetics and radiation; lubrication and surface phenomena.

Space and Environment Technology Center: Magnetospheric, auroral and cosmic ray physics, wave-particle interactions, magnetospheric plasma waves; atmospheric and ionospheric physics, density and composition of the upper atmosphere, remote sensing using atmospheric radiation; solar physics, infrared astronomy, infrared signature analysis; effects of solar activity, magnetic storms and nuclear explosions on the earth's atmosphere, ionosphere and magnetosphere; effects of electromagnetic and particulate radiations on space systems; space instrumentation; propellant chemistry, chemical dynamics, environmental chemistry, trace detection; atmospheric chemical reactions, atmospheric optics, light scattering, state-specific chemical reactions and radiative signatures of missile plumes, and sensor out-offield-of-view rejection. 
SCFEEN IMAGE USER=\#EEE SESSTON-TEOEFOS

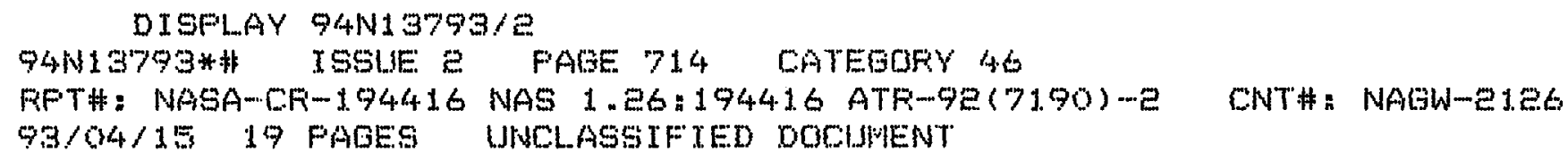

Seg also $A 93-30015$

UTTL: Stomtine transport af ring current and radiation belt iong

AUTH: A/CHEN, MAFGARET W. E/SCHLIZ, MICHAEL: C/LYONS, L. Ri, D/GOFNEY, DAVIL I.

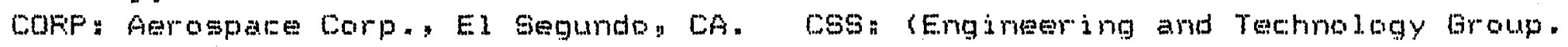
)

SAP: AVAII: CASI HC AOBMF AOL

CIO: UNITED STATES

MATS: / *DIFFISION COEFFICIENT/*EARTH MAGNETOSFHEFE/*FARTICLE HOTION/*FADIATION BELTS / FFING CURFENTS

MINS: I ION MOTION/ IONOSFHERIC DRIFT/ IONDSFHERTC STOKMS

AEA: Auther (revised)

ABS: This is an investigarion of storntime particle transport that laads to formation of the ring current. Bur method is to trace the guiding-center motion of representative ions (having selected first adiabatic inveriants mu) in response to model. substorm-associated impulses in the comvection electrie field. We compare our sinulaticon resultes qualitatively with existing analytically tractable jdealizations of particle transport ENTEF: (mirect convective access and radjal diffusjon) in arder bo assest the 



\section{SCREEN IMAEE USEF=*EEG SESSION=TEOBROS $12 / 14 / 93-02: 58: 17-F H$}

\section{DISFLAY $94 N 13793 / 2$}

limits of validity of these appoximations. For mu appronimately less than $10 \mathrm{MeV} / \mathrm{g}$ (E appraximately less than 10 keV at L equivalent to 3 ) fhe jon drift periad an the fingl. (rimg-current) drift shell of interest (L. equivalent to 3 ) exceeds the duraticon of the main phase of our model. storm. and we find that the tramsport of ions to this drift shell is appropriately idealized as direct convective access, typicaliy from apen d)ift paths. Ion transport to a final closed drift path from an apen (plasma-gheet) drift trajectory is possible for thase portions af that drift path that lie outside the mean stormtime separatrin betwen closed and open drift trajectories. For mu approximately 10-es MeV/G (110 keV approximately less than E appraximately less than 280 keV at L equivalent. to 3) the drift periad at $L$ equivalent to 3 is comparable to the postulated 3-hr duretion of the storn, and the mode of transport is transitional between direct convective access ard transport that resembles radial diffusion. (This particle population is transitional between the ring current and radiation belt) "For mu approsimately greater than es MeV/G (radiation-belt ians having $E$ appreximately greater than ebo keV at: L. equivalent to 3) the ian drift period is considerably shorter than the main phase of a typical storm, and ions gain access to the ring-current region essentialjy via radial diffusion. Ey computing the mean and mean-square cumulative changes in $1 / L$ ameing (in this case) de ENTEF: representative ions equaliy spaced in drift time around the steady-state MOFE 

SCFEEN IMASE USER=*EEB SESSION=TEOEROS 1E/14/93-02:S3:4E-PM

DISFLAY 94M13793/2

drift shell of interest (L. equivalent: to 3 ), we have estimated (firom bath our forward and our time-reversed simulations) the time-integrated rabial-diffusion coefficients D(sup sim) (sub LL) for particles having sejected values of mu approximatejy greater than 15 Mevig. The results.

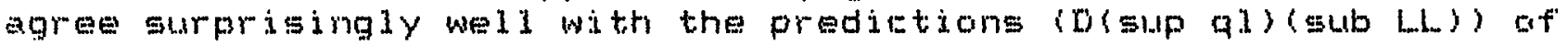
quasilinear radial diffusion theory, despite the rather brief duration (approximately 3 hrs) of our model storm and despite the extreme variability (with frequency) of the spectral-density functicu that characterizes the applied electrit find during our madel storm. $A=$ expected, the values of $D(s u p=j m$ ) (sub $L L$ ) deduced (respectively) from cur forward and time-reveresed simulationg agree even better with each bither and with $D$ (sup $g i m$ ) (sub $L L$ ) when the impulse amplitudes which characterize the individual substorms of our model storm are systematirally reduced. 

Aerospace Report No.

ATR-92(7190)-2

\section{STORMTIME TRANSPORT OF RING CURRENT AND RADIATION BELT IONS}

\section{Prepared by}

M. W. Chen, M. Schulz, L. R. Lyons, and D. J. Gorney Space and Environment Technology Center

Technology Operations

15 April 1993

Engineering and Technology Group THE AEROSPACE CORPORATION

El Segundo, CA 90245-4691

Prepared for

NASA HEADQUARTERS

Washington, DC 20546

Grant No. NAGW 2126

PUBLIC RELEASE IS AUTHORIZED 

Aerospace Report No. ATR-92(7190)-2

STORMTIME TRANSPORT OF RING CURRENT AND RADIATION BELT IONS

Prepared

Margaret Chen M. W. Chen

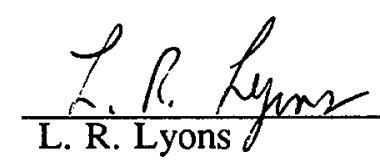

Approved

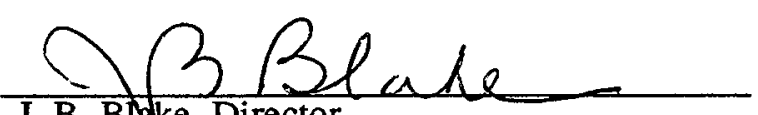

J. B. Bit ae, Director

Space Particles and Fields Department


D. J. Gorey

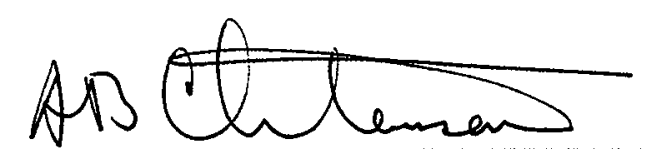

A. B. Christensen, Principal Director Space and Environment Technology Center

PRECEDING PAGE BLANK NOT FHLMELB

RAGE $\dot{H}^{\prime}$

iii

INTENTIONAL:

Darin 

NOTE

The material reproduced in this report originally appeared in the Journal of Geophysical Research. The ATR is published to document the work for the corporate record. 



\title{
Stormtime Transport of Ring Current and Radiation Belt Ions
}

\author{
Margaret W. Chen, Michael Schulz, LaRRy R. Lyons, and David J. GorNeY \\ Space and Environment Technology Center, The Aerospace Corponation, El Segundo, California
}

\begin{abstract}
This is an investigation of stormtime particle transport that leads to formation of the ring current. Our method is to trace the guiding-center motion of representative ions (having selected first adiabatic invariants $\mu$ ) in response to model substorm-associated impulses in the convection electric field. We compare our simulation results qualitatively with existing analytically tractable idealizations of particle transport (direct convective access and radial diffusion) in order to assess the limits of validity of these approximations. For $\mu \lesssim 10 \mathrm{MeV} / \mathrm{G}(E \lesssim 110 \mathrm{keV}$ at $L \approx 3)$ the ion drift period on the final (ring-current) drift shell of interest $(L \approx 3)$ exceeds the duration of the main phase of our model storm, and we find that the transport of ions to this drift shell is appropriately idealized as direct convective access, typically from open drift paths. Ion transport to a final closed drift path from an open (plasma-sheet) drift trajectory is possible for those portions of that drift path that lie outside the mean stormtime separatrix between closed and open drift trajectories. For $\mu \sim 10-25 \mathrm{MeV} / \mathrm{G}(110 \mathrm{keV} \lesssim E \lesssim 280 \mathrm{keV}$ at $L \approx 3)$ the drift period at $L \approx 3$ is comparable to the postulated 3-hr duration of the storm, and the mode of transport is transitional between direct convective access and transport that resembles radial diffusion. (This particle population is transitional between the ring current and radiation belt). For $\mu \gtrsim 25 \mathrm{MeV} / \mathrm{G}$ (radiation-belt ions having $E \gtrsim 280 \mathrm{keV}$ at $L \approx 3$ ) the ion drift period is considerably shorter than the main phase of a typical storm, and ions gain access to the ring-current region essentially via radial diffusion. By computing the mean and mean-square cumulative changes in $1 / L$ among (in this case) 12 representative ions equally spaced in drift time around the steady-state drift shell of interest $(L \approx 3)$, we have estimated (from both our forward and our time-reversed simulations) the time-integrated radial-diffusion coefficients $D_{L L}^{\text {sim }}$ for particles having selected values of $\mu \gtrsim 15$ $\mathrm{MeV} / \mathrm{G}$. The results agree surprisingly well with the predictions $\left(D_{L L}^{\mathrm{q}}\right)$ of quasilinear radialdiffusion theory, despite the rather brief duration $(\approx 3 \mathrm{hr})$ of our model storm and despite the extreme variability (with frequency) of the spectral-density function that characterizes the applied electric field during our model storm. As expected, the values of $D_{L L}^{\text {sim }}$ deduced (respectively) from our forward and time-reversed simulations agree even better with each other and with $D_{L i}^{\text {sim }}$ when the impulse amplitudes which characterize the individual substorms of our model storm are systematically reduced.
\end{abstract}

\section{INTRODUCTION}

This work is an outgrowth of our efforts to understand in detail the magnetospheric charged-particle transport that leads to stormtime ring-current formation. The ring current consists of geomagnetically trapped ions and electrons in the 10-200 keV energy range [e.g., Frank, 1967; Williams, 1981], and its global intensity is commonly measured by the geomagnetic index $D_{\text {st }}$ [e.g., Mayaud, 1980, pp. 115-129]. The quiet-time ring current is believed [e.g., Hamilton et al., 1988] to contribute about $10-20 \mathrm{nT}$ to $-D_{\text {st }}$, but this is largely offset by magnetopause currents during the geomagnetically quiet intervals which define the baseline $\left(D_{s t}=0\right)$ for the index. Thus, the increase in $\left|D_{s t}\right|$ to $200 \mathrm{nT}$ typically observed during the main phase of a major geomagnetic storm must correspond [cf. Dessler and Parker, 1959; Sckopke, 1966] to perhaps a tenfold or twentyfold increase in the energy content of the trapped-particle population.

Indeed, major increases in trapped-electron [Williams and Smith, 1965; Pfitzer et al., 1966; Craven, 1966; Söraas and Davis, 1968; Bostrom et al., 1970; Lyons and Williams, 1975] and in trapped-ion [Frank, 1967; Frank and Owens, 1970; Smith and Hoffman,

Copyright 1993 by the American Geophysical Union.

Paper number 92JA02608.

0148-0227/93/92JA-02608\$05.00
1973; Williams and Lyons, 1974; Lyons and Williams, 1976; Williams, 1981; Lui et al., 1987] fluxes are known to occur in connection with major geomagnetic storms. Such increases actually extend from $L \sim 7$ to as low as $L \sim 2$ and span particle energies from $\lesssim 1 \mathrm{keV}$ to several hundred $\mathrm{keV}$. However, the main contribution to $\left|D_{s t}\right|$ comes from particles in the $10-200 \mathrm{keV}$ energy range, since these contribute the overwhelming majority of the energy content of geomagnetically trapped particles. Trapped-particle enhancements at $L \gtrsim 4$ are commonly associated with isolated substorms, which. can occur during otherwise quiet intervals, but the "injection" of ions and electrons to $L \lesssim 4$ corresponds to a geomagnetic storm, and particle injection to $L \lesssim 3$ corresponds to a major storm. Since particles injected to $L \lesssim 4$ are presumed to be the ones mainly responsible [Akasofu, 1963; Frank, 1967] for storm-associated increases in $\left|D_{s t}\right|$, we must account for these flux increases in order to understand geomagnetic storms.

Formation of the stormtime ring current presumably can be understood in terms of the transport of charged particles within the magnetosphere by storm-associated variations in the convection electric field. Early studies of charged-particle motion in the magnetosphere [e.g., Kavanagh et al., 1968; Chen, 1970] took into account the effects of a steady-state convection electric field. Chen [1970] described the adiabatic drift paths of equatorially mirroring low-energy protons in a dipolar magnetic field, with a radial corotation electric field and 
a uniform steady-state convection electric field superimposed. Later Ejiri [1978] traced particle trajectories under the influence of an increased steady-state convection electric field for arbitrary pitch angles. These models were able to account for substorm-associated enhancements of 1-100 keV particles observed near the plasmapause by Explorer 45.

Although such steady-state descriptions are useful in explaining gross features of particle transport, temporal variations in the convection electric field must play an important role in both the injection and the trapping of particles during storms. Using a numerical model, Roederer and Hones [1974] discussed the characteristics of a time-varying electric field that could explain the frequent ATS observations [DeForest and McIlwain, 1971] of plasma injection into the equatorial magnetosphere. They found that low-energy particles $(\lesssim 10 \mathrm{keV}$ ) injected earthward from the tail by enhanced storm-associated electric fields can become trapped on closed drift paths when the enhanced electric field decays away. Smith et al. [1979] illustrated this effect by tracing the motion of equatorial particles that had been injected from $L=10$ on the night side in a dipolar field model. Later, Takahashi [1990], using the more realistic magnetic field model of Tsyganenko [1987], made a similar tracing of particle drifts. Injection onto closed drift paths thus results directly from enhanced particle convection.

The consequences of transporting low-energy $(\lesssim 40$ $\mathrm{keV}$ ) ring-current particles inward during a storm were discussed by Lyons and Williams [1980]. By mapping phase-space densities in accordance with Liouville's theorem, they showed that enhanced trapped-particle fluxes observed at $L<4$ during two large storms could be accounted for by invoking a 1-3 $R_{E}$ inward radial displacement of the previously-trapped particle distribution under conservation of the first two adiabatic invariants. The associated inward transport to a region of stronger magnetic field strength would result in the requisite particle energization. Assuming a duration $\sim 3 \mathrm{hr}$ for the main phase of a storm, they found that this "displacement" would have required a mean enhancement $\sim 1 \mathrm{mV} / \mathrm{m}$ in the azimuthal component of the convection electric field across the nightside magnetosphere. Particles having drift periods much longer than the main phase of the storm might then remain at a nearly constant local time $(\phi)$ so that the enhanced convection electric field could transport them uniformly inward. This model for particle transport into the ring-current region is called "direct convective access" [Lyons and Williams, 1980]. It applies to low-energy particles ( $\lesssim 40 \mathrm{keV}$ ) and represents a limiting case for which the particle kinematics can be solved analytically. Particles with energies $\gtrsim 40 \mathrm{keV}$ would gradient-drift significantly in $\phi$ during a 3 -hr main phase and so would not satisfy the constant- $\phi$ approximation.

It is estimated, however, that $50-75 \%$ of the stormtime ring current energy is associated with particle energies $\gtrsim 40 \mathrm{keV}$ [Williams, 1980]. Thus, an understanding of the stormtime transport mechanism for higher-energy particles is at least equally important. Lyons and Schulz [1989] have recently treated the inward transport of $\gtrsim 40 \mathrm{keV}$ particles by using radial- diffusion theory as a first approximation. Using the random-impulse model of Cornwall [1968] to generate the fluctuation spectrum required in the resonant-particle diffusion theory of Fälthammar [1965], they found that storm-associated equatorial convection electric field fluctuations having root-mean-square magnitudes typical of those observed $(\sim 1.0-1.5 \mathrm{mV} / \mathrm{m})$ could adequately account for the increased trapped-particle fluxes typically observed at $2 \lesssim L \lesssim 4$ during the main phase of a storm. Although radial-diffusion theory is an idealization of doubtful validity for ring-current particle energies $\lesssim 100 \mathrm{keV}$, it represents a second limit which is analytically tractable.

Direct convective access and radial diffusion are thus two limits that have been treated analytically and invoked to account for the enhanced ring-current particle fluxes observed at $2 \lesssim L \lesssim 4$ during main phases of storms. However, much of the energy content of the ring current lies in an intermediate energy range $(\sim 40-$ $100 \mathrm{keV}$ ) for which the assumptions invoked in order to arrive at these simplified analyses of the transport process are not valid. At such intermediate energies, the particle drift period can be comparable to the duration of the main phase of a storm, and so it is not clear how to describe the transport mechanism except via guiding-center simulations.

We have undertaken such a guiding-center simulation to elucidate the process of stormtime ion transport and to assess the limits of validity of direct convective access and radial diffusion approaches. Our study entails certain innovations which facilitate the analysis of our results.

1. We have synthesized an ensemble of model geomagnetic storms, each consisting of almost randomly occurring exponentially decaying electrostatic impulses with quasi-random onset times. Since we express our electric field in analytical form, our approach enables us to make exhaustive diagnostic analyses on the consequences of each model storm in the ensemble.

2. We have taken care to arrange our representative test particles isochronally (i.e., at equal spacings in elapsed quiescent drift time rather than, for example, at equal spacings in magnetic longitude) around each drift shell of interest at the beginning of each simulation.

We have found that the stormtime access of ions having $E \lesssim 110 \mathrm{keV}$ to drift shells of interest in the context of ring-current formation is achieved typically by convection from the nightside neutral line along open drift trajectories. The minimum time required for this convective transport to occur is well approximated by generalizing the estimate of Lyons and Williams [1980] to the field model used in our study. The fraction of representative particles that gain access by direct convection to the drift shell of interest decreases with increasing ion energy, to the extent that particles having $E \gtrsim 165 \mathrm{keV}$ arrive almost exclusively from previously closed drift trajectories of either larger or smaller $L$ value. For these particles we found that a transport description based on radial-diffusion theory is appropriate.

Indeed, for $\mu \gtrsim 15 \mathrm{MeV} / \mathrm{G}$ which corresponds to $E \gtrsim 165 \mathrm{keV}$ at $L \sim 3$ we have found interesting relationships upon comparing the radial-diffusion coeffi- 
cients $D_{L L}$ deduced from our simulation results with the quasilinear predictions of Fälthammar [1965] and Cornwall [1968]. In particular, we have found increasingly good agreement (even for an individual model storm having a 3-hr main-phase duration) between our simulation results and the quasilinear predictions of Fälthammar [1965] upon decreasing the amplitudes of the electric field impulses in our ensemble of model storms. The quasilinear diffusion coefficient for an individual storm is a remarkably variable function of the first adiabatic invariant $\mu$, and we have found that Fälthammar's quasilinear theory accounts surprisingly well for the $\mu$ values at which our simulation yields especially small values for $D_{L L}$. Despite these encouraging numerical results, the use of quasilinear diffusion theory to describe radial transport is marginally justified from a fundamental standpoint at energies ( $\lesssim 165 \mathrm{keV}$ ) primarily responsible for the ring current. Thus, we have extended our simulations and our comparisons into the higher-energy radiation-belt range (ultimately to $\mu=$ $250 \mathrm{MeV} / \mathrm{G}$, which corresponds to $E=2.75 \mathrm{MeV}$ at $L=3$ ).

\section{FIELD MODEL}

The magnetic field model used in this model is obtained [cf. Dungey, 1961; Hill and Rassbach, 1975] by adding a uniform southward field $\Delta \mathbf{B}$ to the geomagnetic dipole field. We invoke this simple field configuration because it enables us to make direct comparisons between the simulated transport and previous analytical formulations. An advantage of our model over a purely dipolar field is the presence of a quasimagnetopause at the boundary between closed and open field lines (see Figure 1). The equation of a field line in this model is

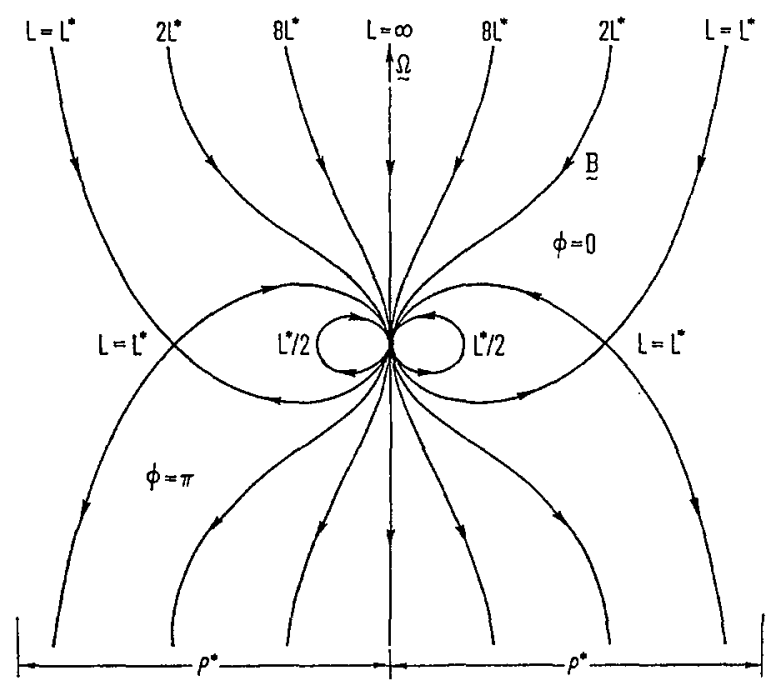

Fig. 1. An illustration of the magnetospheric magnetic field mode used in this study. The model is symmetric about the $\sin \theta=0$ axis and about the equatorial $(z=0)$ plane, which contains a circular neutral line at $r=b$ on the magnetic shell $L=L^{*}$. This de fines a quasi-magnetopause which approaches an asymptotic distance $\rho^{*}$ from the tail axis at large distances $|z|$ from the equatorial plane.

$$
\left[1+0.5\left(\frac{r}{b}\right)^{3}\right]^{-1}\left(r / R_{E}\right) \csc ^{2} \theta=\text { const } \equiv L
$$

where $r$ is the geocentric distance, $\theta$ is the magnetic colatitude, $R_{E}$ is the radius of the Earth, and $b=$ $1.5 L^{*} R_{E}=12.82 R_{E}$ is the radius of the equatorial neutral line. This value of $b$, which is obtained by mapping the last closed field line (denoted $L^{*}$ ) to a colatitude of $20^{\circ}$ on the Earth, corresponds to $|\Delta B|=14.474 \mathrm{nT}$ and $L^{*}=8.547$. The limit $b \rightarrow \infty\left(L^{*} \rightarrow \infty\right)$ would correspond to a purely dipolar $\mathbf{B}$ field. In this study, we consider only equatorially mirroring particles. Our equatorial field intensity $B_{0}$ is given by

$$
B_{0}=\left(\frac{\mu_{E}}{r^{3}}\right)-14.474 \mathrm{nT}
$$

where $\mu_{E}=3.05 \times 10^{4} \mathrm{nT}-R_{E}^{3}$ is the geomagnetic dipole moment. Further details of this field model are given by $S \operatorname{chulz}[1991$, pp. 98-110].

We assume that the total electric field $\mathbf{E}=-\nabla \Phi_{E}$ is derivable from the scalar potential

$$
\Phi_{E}=-\frac{V_{\Omega}}{L}+\frac{V_{0}}{2}\left(\frac{L}{L^{*}}\right)^{2} \sin \phi+\frac{\Delta V(t)}{2}\left(\frac{L}{L^{*}}\right) \sin \phi,
$$

in which the three separate terms correspond to corotation $\left(V_{\Omega}=90 \mathrm{kV}\right)$, the Volland-Stern [Volland, 1973; Stern, 1973] model of quiescent convection $\left(V_{0}=50\right.$ $\mathrm{kV}$ ), and the time-dependent enhancement $\Delta V(t)$ associated with the stormtime convection, respectively. The time-varying term in the potential is assumed to vary as $L$ [cf. Nishida, 1966; Brice, 1967] rather than as $L^{2}$ because electric disturbances are expected to be less well shielded than steady-state convection by the inner magnetosphere.

Whereas others have imposed time-varying crossmagnetospheric electric fields either estimated from $K p$ [e.g., Smith et al., 1979; Kistler et al., 1989] or deduced (insofar as possible) from direct observations [Riley and Wolf, 1992] during particular storms, we have instead synthesized an ensemble of model geomagnetic storms. In our model the storm-associated enhancement $\Delta V(t)$ in the cross-tail potential drop

$$
\Delta V(t)=\sum_{i=1}^{N} \Delta V_{i} \exp \left[\left(t_{i}-t\right) / \tau\right] \theta\left(t-t_{i}\right)
$$

where $\theta(t)$ is the unit step function ( $\equiv 1$ for $t \geq 0 ; \equiv 0$ for $t<0$ ), as a superposition of almost randomly occurring impulses that rise sharply and decay exponentially with a "lifetime" $\tau=20 \mathrm{~min}$ [cf. Cornwall, 1968]. The impulses represent the constituent substorms of a storm. The potential drop $\Delta V_{i}$ associated with any impulse is chosen randomly from a Gaussian distribution with a $200-\mathrm{kV}$ mean and a $50-\mathrm{kV}$ standard deviation. Negative impulses (such that $\Delta V_{i}<0$ ) are thus theoretically possible but extremely improbable, corresponding to only $0.003 \%$ of all impulses. We have chosen such a large mean for the $\Delta V_{i}$ since our intention is to model a major $\left(\left|D_{s t}\right| \sim 200 \mathrm{nT}\right)$ storm, such as those which Lyons and Williams [1980] analyzed. Since those storms had a main phase lasting $\sim 3 \mathrm{hr}$, we assume 
here that the $N$ start times $t_{i}$ in (4) are randomly distributed within a 3 -hr time interval which corresponds to the main phase of our model storm. However, we impose a 10-min "dead time" (after each impulse onset) during which no subsequent impulse can start. This constraint imposes a realistic delay between the occurrences of consecutive impulses and thus leads to a reasonable distribution of impulses. Without such a dead time it would be possible for the next impulse to start immediately after the previous one. We found (see Figure $2 a$ ) that this could result in the buildup of unrealistically large cross-tail potentials, and so we rejected the model in which the $t_{i}$ in (4) were randomly distributed without dead time. The technical details of how we determine the start times of the impulses are given in the appendix.

We have constructed 100 such random storms so that on average there are $\bar{N}=9$ impulses per storm or 3 substorms/hr. We have randomly chosen one model storm for a detailed case study. Figure $2 b$ shows the variation in cross-tail potential for this prototypical storm. The mean cross-tail potential drop over the time interval $t_{1}<t<t_{1}+3 \mathrm{hr}$ in this case is $230 \mathrm{kV}$, of which $50 \mathrm{kV}$ corresponds to $V_{0}$ and $180 \mathrm{kV}$ to $\langle\Delta V(t)\rangle$. This is a fairly typical value for $\langle\Delta V(t)\rangle$ among the 100 storms that we constructed since the ensemble-averaged $\langle\Delta V(t)\rangle$ turned out to be $184 \mathrm{kV}$.

\section{PARTICLE DYNAMICS}

Since we are simulating the guiding-center motion of nonrelativistic equatorially mirroring particles here, we treat the first two adiabatic invariants $(\mu \neq 0$ and $J=0$, respectively) as conserved quantities. In this case, the guiding-center Hamiltonian $H$ is equal to the total energy (kinetic plus potential) of a particle. This is given by

$$
H=\mu B_{0}+q \Phi_{E},
$$

where $q$ is the charge of the particle. It follows from $(2)-(4)$ and from Hamilton's equations of motion [e.g.,

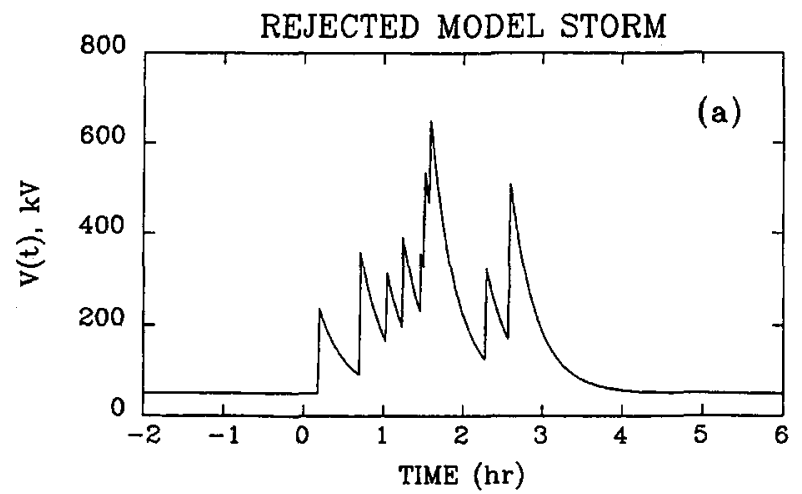

Goldstein, 1980, p. 339] that the guiding-center motion of an equatorially mirroring particle subject to $\mathbf{E} \times \mathbf{B}$ and gradient- $B$ drifts is described by the equations

$$
\frac{d L}{d t}=\frac{L^{2} R_{E} \cos \phi}{2 \mu_{E}}\left[V_{0}\left(\frac{L}{L^{*}}\right)^{2}+\Delta V(t)\left(\frac{L}{L^{*}}\right)\right]
$$

and

$$
\begin{aligned}
\frac{d \phi}{d t}= & \Omega-3 \frac{\mu \mu_{E}}{q B_{0} r^{5}} \\
& -\frac{R_{E}}{\mu_{E}}\left[V_{0}\left(\frac{L}{L^{*}}\right)^{2}+\frac{\Delta V(t)}{2}\left(\frac{L}{L^{*}}\right)\right] L \sin \phi
\end{aligned}
$$

where $\Omega$ is the angular velocity of the Earth and $\phi$ is the azimuthal coordinate (local time). We neglect particle loss processes such as charge exchange and Coulomb drag in this study.

We solve the ordinary differential equations (6) and (7) simultaneously by using the Bulirsh-Stoer extrapolation method with variable time step [e.g., Press et al., 1986, pp. 563-568] for specified initial conditions. First, by setting $\Delta V(t)=0$ in (6) and (7), we obtain steady-state adiabatic drift paths associated with a particular value of the first adiabatic invariant $\mu$. We start 12 representative particles at points equally spaced in time on a steady-state drift path. We then apply a storm-associated tempóral variation $\Delta V(t)$ in the crosstail potential drop and run the simulation to determine the consequent stormtime particle transport. We can run the simulation either backward in time (to determine where any representative particle must have been prior to the storm in order to reach the desired phase on its "final" drift shell) or forward in time (to follow the dispersal of initially codrifting particles among drift shells during and after the storm.)

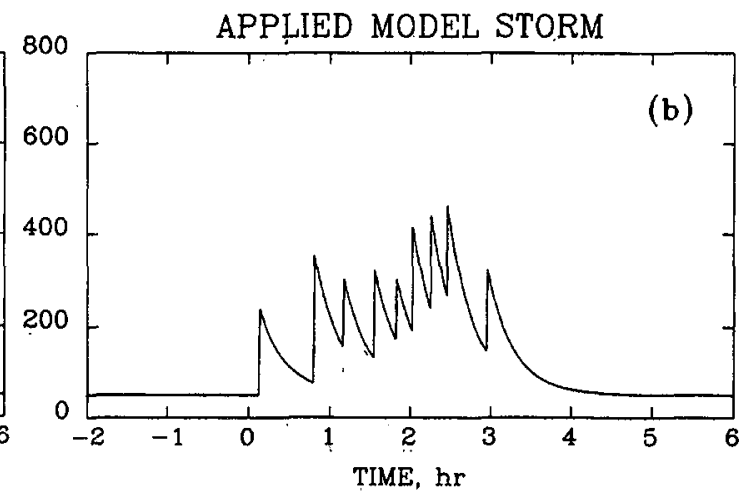

Fig. 2. (a) We have rejected the storm model in which the onsets of superimposed impulses in the cross-tail potential occur purely at random. Without any realistic delay (i.e., dead time) between the onsets of consecutive impulses, it is possible to attain an unrealistically large cross-tail potential (e.g., $\sim 650 \mathrm{kV}$ at $t \approx 1.6 \mathrm{hr}$ in this instance). (b) We have adopted and applied a model storm in which the cross-tail potential is enhanced by a superposition of exponentially decaying impulses (decay time $\tau=20 \mathrm{~min}$ ). These represent the constituent substorms of a storm and start at times which are distributed randomly over a 3-hr timè interval (main phase) except that we impose, after the start of each impulse, a 10-min "dead time" during which no subsequent impulse can start. 
The maximum time step in our simulations is restrained so as to ensure good accuracy. We have tested our results against the conservation law [cf. Goldstein, 1980, p. 348]

$$
\frac{d H}{d t}-\frac{\partial H}{\partial t}=\frac{\partial H}{\partial R} \frac{d R}{d t}+\frac{\partial H}{\partial \phi} \frac{d \phi}{d t}=0
$$

on selected representative particles for time-dependent $\Delta V(t)$ as well as for $\Delta V(t) \equiv 0$ and have found (8) to be well satisfied throughout our simulations.

\section{Simulated TraJectories}

In this section we present results of the simulated transport of singly charged ions having various $\mu$ values and vanishing second invariant $(J=0)$. The dashed outer circle (shown in whole or in part on each particletrajectory plot) indicates the location of the neutral line, a circle of radius $R=12.82$, which marks the boundary between open and closed magnetic field lines in our magnetic-field model (cf. Figure 1). The lefthand panels in Figures 3-4 show steady-state ion drift paths in the equatorial plane for selected $\mu$ values. For $\mu \lesssim 26.5 \mathrm{MeV} / \mathrm{G}$ there is an $\mathrm{x}$-type stagnation point associated with the separatrix between open and closed drift trajectories.

Figure $3 a$ is for $\mu=3 \mathrm{MeV} / \mathrm{G}$, which corresponds to a kinetic energy of $33 \mathrm{keV}$ at $R \equiv r / R_{E}=3$. The ionic drift for this $\mu$ value has its stagnation point at $R=$ 4.4 on the dawn meridian. We label closed drift shells in terms of the dimensionless third adiabatic invariant defined by Roederer [1970, p. 107] as

$$
L \equiv\left|\frac{2 \pi \mu_{E}}{\Phi_{B} R_{E}}\right|=\left[\frac{1}{2 \pi} \int_{0}^{2 \pi} \frac{d \phi}{L(\phi)}\right]^{-1},
$$

where $\Phi_{B}$ is the magnetic flux enclosed by that drift shell and $L(\phi)$ denotes the field-line label defined by (1) at longitude $\phi$ on the drift shell. For example, the drift shell highlighted by the small solid circles in Figure $3 a$ bears the label $L=3.14$.

The right-hand panels in Figure 3-4 illustrate the stormtime access of particles to the drift path which crosses the dusk meridian at $R=3$. This drift path has been chosen as representative of the particles that are primarily responsible [cf. Akasofu, 1963; Frank, 1967] for storm-associated increases in the ring-current index $\left|D_{s t}\right|$. The steady-state drift period $\left(\tau_{3}=12.0 \mathrm{hr}\right)$ for ions with $\mu=3 \mathrm{MeV} / \mathrm{G}$ on such a path is 4 times the duration of our model storm. "Starting" with 12 representative particles (as indicated by the solid circles) equally spaced in time on such a drift path, we have applied our model storm (see Figure $2 b$ ) and have run the simulation backward in time so as to determine where the corresponding ions must have been located prior to the storm in order to have been transported to their final drift phases on the steady-state drift path in question. We followed open trajectories backward in time to the neutral line and closed trajectories backward in time for at least one quiescent drift period prior to the onset of the first impulse. To ensure that the last impulse has decayed by at least nine $e$-foldings, we initiate the time-reversed simulation at $t=t_{1}+3 \mathrm{hr}$ $+(m / 12) \tau_{3}$ where $\tau_{3} \geq 3 \mathrm{hr}$ and $m$ is an integer. We impose this last condition in a $\tau_{3}$-dependent way simply to make the representative ions' phases correspond approximately to those shown in Figures 3-4. Since ions which differ in drift phase respond differently to the model storm, the right-hand panels of Figures 3-4 illustrate (on an expanded scale) the range of possible responses.

The small solid circles, spaced $30^{\circ}$ apart in drift phase, in Figure $3 b$ correspond to those in Figure $3 a$ and thus mark the final steady-state drift path. Eight of the 12 representative particles could have been transported inward from the night side to populate the final closed drift path. Only one of these 8 representative ions would have been located at $L<L^{*}=8.547$ at storm onset $(t=0)$; its position at that time is indicated by the open circle. The other 7 of these 8 would have crossed the neutral line at times $t>0$. Their access to the ring-current region is essentially convective [cf. Lyons and Williams, 1980]. Four of the 12 representative ions would have been transported outward to the drift shell of interest $(L=3.14)$ from closed drift shells of smaller $L$.

Superposition of the mean stormtime separatrix (dashed-dotted curve) on the stormtime drift paths elucidates the condition for direct convective access to the drift shell of interest and further justifies the inference that this is the main mode of transport to the $L=3.14$ drift shell for $\mu=3 \mathrm{MeV} / \mathrm{G}$. The mean stormtime separatrix is obtained by setting $V_{0}=50 \mathrm{kV}$ and $\Delta V(t) \equiv\langle\Delta V(t)\rangle=180 \mathrm{kV}$ in (3). It represents the boundary between open and closed adiabatic drift paths during the storm. Eleven of the 12 representative ions on the final steady-state drift path of interest lie outside the region enclosed by the stormtime separatrix, and so we might expect approximately this fraction to have direct access to it during the storm. This is only a rough statistical prediction, however, and in fact our simulation showed that only 8 of the 12 representative particles gain access in this way.

The case $\mu=10 \mathrm{MeV} / \mathrm{G}$ corresponds to an ion energy of $110 \mathrm{keV}$ at $R=3$. Steady-state ion drift paths in the equatorial plane for this case are shown in Figure $3 c$. The $\mathrm{x}$-type stagnation point for $\mu=10 \mathrm{MeV} / \mathrm{G}$ is located on the dawn meridian at $R=6.5$, which is farther from the Earth than the stagnation point for $\mu=3 \mathrm{MeV} / \mathrm{G}$. The drift shell that intersects the dusk meridian at $R=3$ corresponds to $L=3.00$ for $\mu=10$ $\mathrm{MeV} / \mathrm{G}$, and the quiescent drift period $\left(\tau_{3}=2.4 \mathrm{hr}\right)$ for these ions has the same order of magnitude as the duration of the storm.

Results of the time-reversed simulation for $\mu=10$ $\mathrm{MeV} / \mathrm{G}$ are shown on an expanded scale in Figure 3d. For this higher value of $\mu$, the model storm has transported fewer particles (only 6 of the 12) inward along open drift paths to the closed drift shell of interest in a manner which resembles direct convective access. The other half had already been on closed drift shells when the storm began, and 5 of these had actually started on drift shells interior to the final drift shell of interest. The final drift period is comparable to the duration of 
the storm in this case, and the transport seems transitional between direct convective access (for half of the representative particles) and something that resembles radial diffusion for the other half.

This point is further illustrated by superimposing the mean stormtime separatrix (dashed-dotted curve, Figure $3 d$ ) on the final steady-state drift path. Because the stormtime separatrix lies slightly earthward of the final steady-state drift path of interest on the dusk side, par-
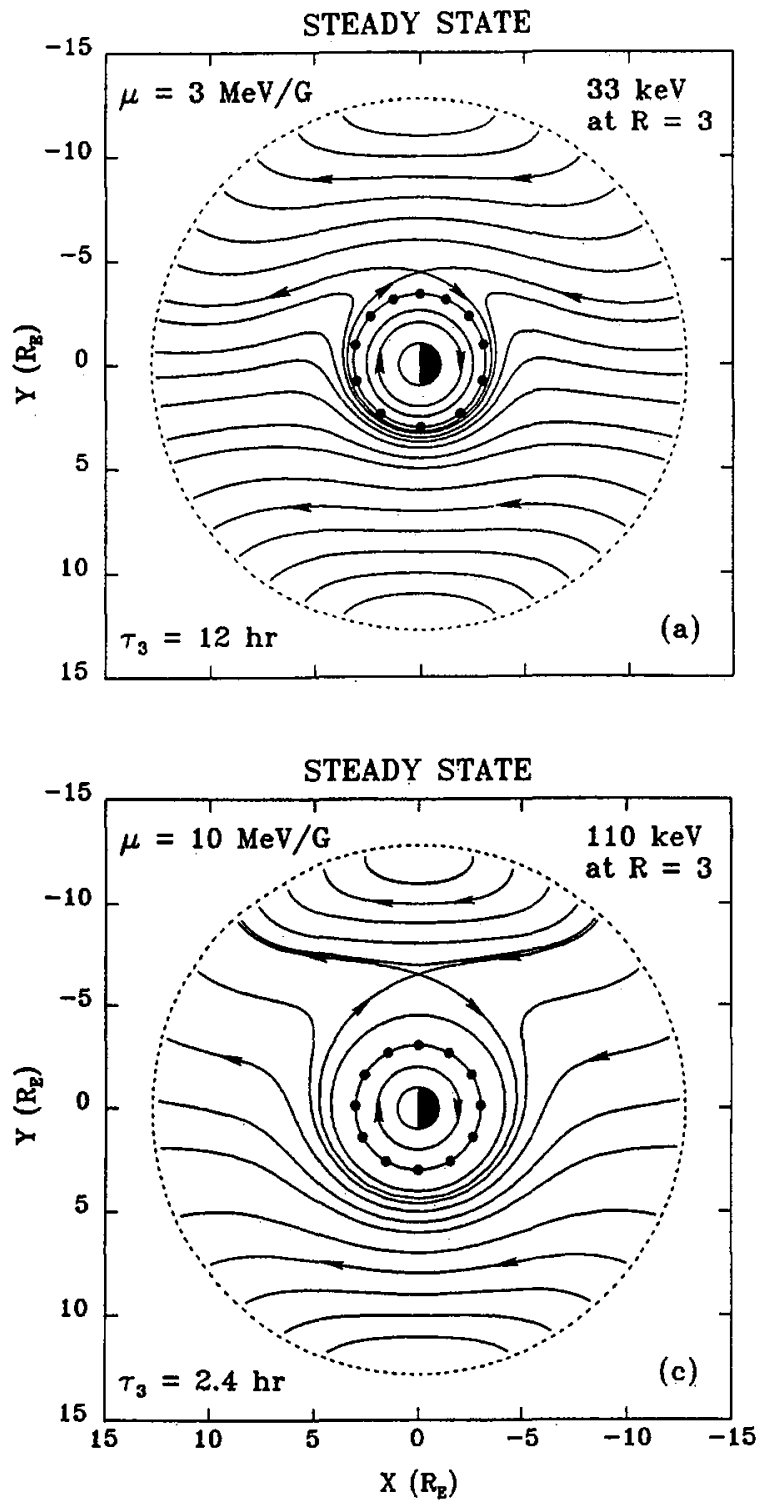

ticles initially on open drift paths that pass near there can gain access during the storm to the region of closed steady-state drift paths. This argument should apply to only about half of the drift shell of interest and so the other half presumably could not have been populated from open drift paths during this model storm. We cannot predict just which representative particles will gain access along open trajectories and which will not. Instead, about half of the $L=3.00$ drift shell
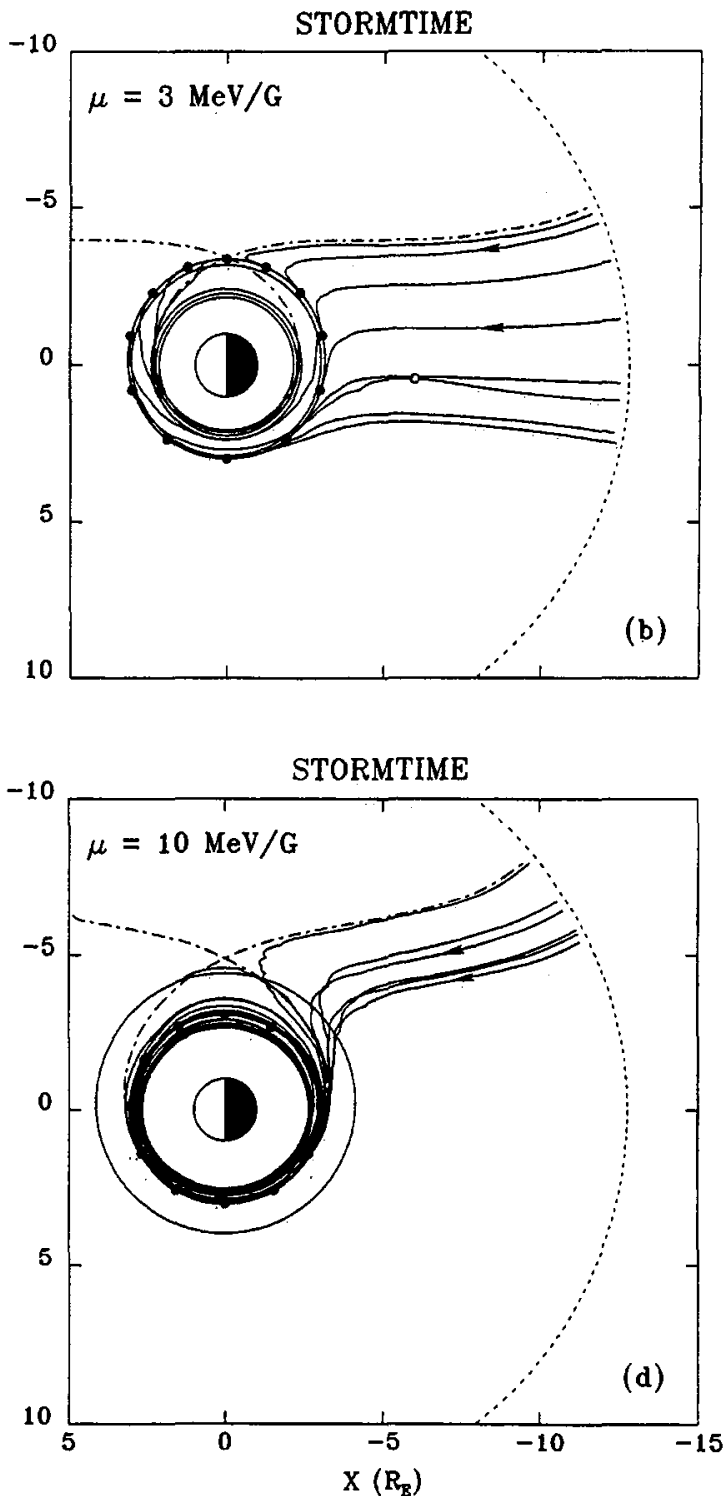

Fig. 3. Quiet-time trajectories of singly charged ions having (a) $\mu=3$ and (c) $10 \mathrm{MeV} / \mathrm{G}$ are plotted in the lefthand panels. The outer dashed circle represents the neutral line at $r=b$. Ions whose drift paths cross the dusk meridian at $R=3$ have drift periods $\tau_{3}=12 \mathrm{hr}$ and $2.4 \mathrm{hr}$ for $\mu=3$ and $10 \mathrm{MeV} / \mathrm{G}$, respectively, as noted. The 12 representative ions' "final" positions (corresponding to the beginning of our time-reversed simulation) on the final steady-state drift path of interest are marked by small solid circles (partially obscured in right-hand panels). The corresponding stormtime trajectories, as computed in our time-reversed simulation, are shown on an expanded scale in the right-hand panels (Figures $3 b$ and $3 d$ ). The dashed-dotted curves represent mean "stormtime" separatrices. The small open circle indicates the "initial" position of the one representative ion that was on an open quiet-time drift trajectory but would have been inside the magnetosphere (i.e., at $L<L^{*}$ ) at storm onset. The dashed portion of the corresponding trajectory applies to times before storm onset $\left(t<t_{1}\right)$. 

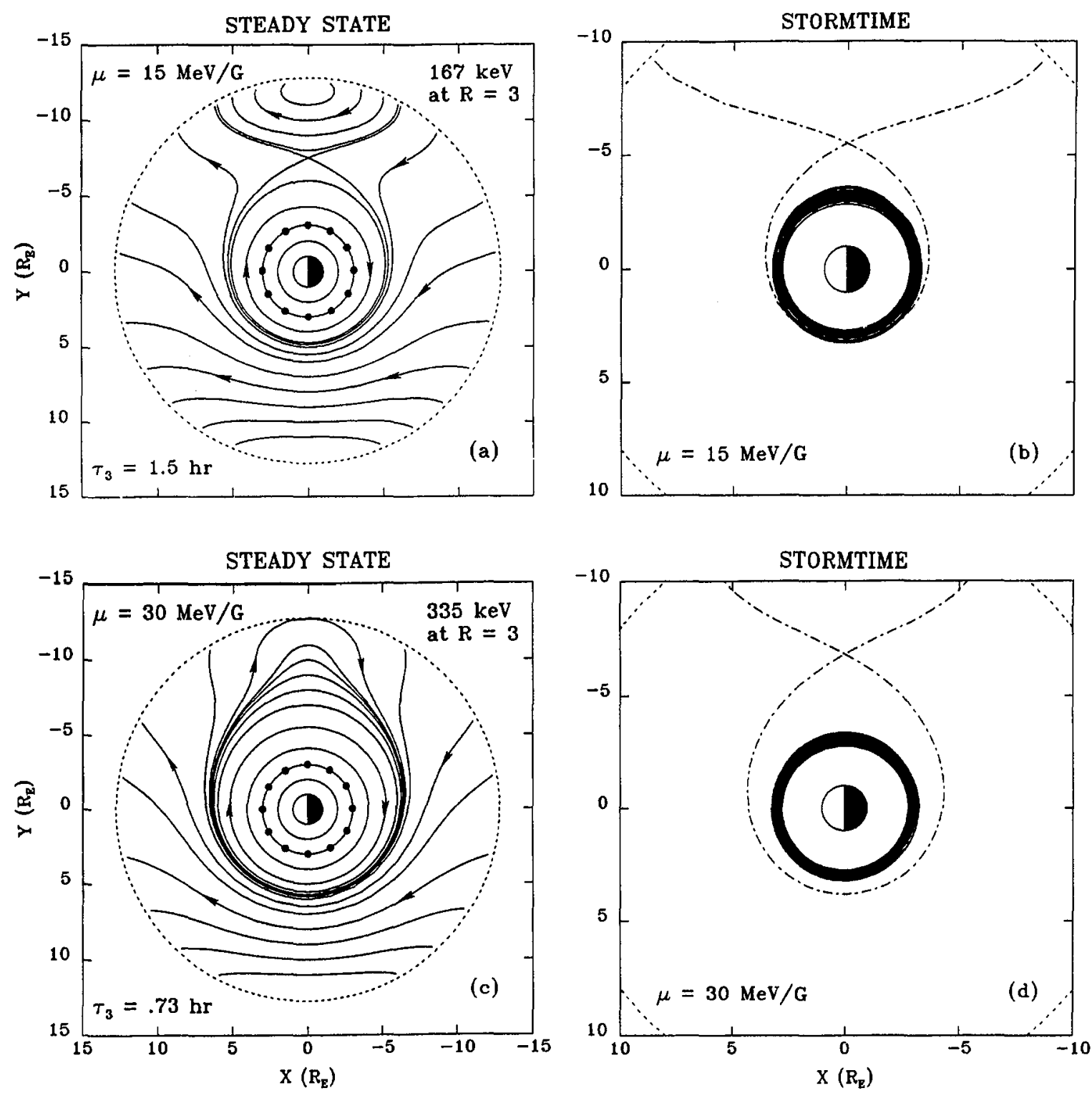

Fig. 4. Quiet-time trajectories of singly charged ions having $(a) \mu=15$ and(b) $30 \mathrm{MeV} / \mathrm{G}$ are plotted in the lefthand panels. Ions which cross the dusk meridian at $R=3$ have drift periods $\tau_{3}=1.5 \mathrm{hr}$ and 0.73 hour for $\mu=15$ and $30 \mathrm{MeV} / \mathrm{G}$, respectively. The 12 representative ions' "final" positions (corresponding to the beginning of our time-reversed simulations) on the final steady-state drift path of interest are marked by small solid circles (totally obscured in right-hand panels). Corresponding stormtime trajectories computed in our time-reversed simulation are shown on an expanded scale in the right-hand panels (Figures $4 b$ and $4 d$ ). The dashed-dotted curves represent the mean stormtime separatrices for the respective $\mu$ values.

for $\mu=10 \mathrm{MeV} / \mathrm{G}$ should be populated by stormtime transport from open drift paths and about half from closed. Our simulation bears out this expectation.

Ions having $\mu=15 \mathrm{MeV} / \mathrm{G}$ would have energies of $167 \mathrm{keV}$ at $R=3$. Steady-state adiabatic drift paths for these (see upper left-hand panel of Figure 4) show a stagnation point at $R=7.4$ on the dawn meridian. This is still farther from the Earth than for $\mu=10$ $\mathrm{MeV} / \mathrm{G}$. Again, the final drift shell of interest is that which intersects the dawn meridian at $R=3$. The quiescent drift period $\tau_{3}$ for these ions is $90 \mathrm{~min}$, which is only half the duration of the storm.

The stormtime trajectories are plotted on an expanded scale in Figure $4 b$. We find all the representative ions to have been transported from closed drift paths, some from smaller and some from larger $L$ values. This leads to a spread among the initial $L$ values. The stormtime transport for $\mu=15 \mathrm{MeV} / \mathrm{G}$ at $L \sim 3$ thus resembles radial diffusion although this was a time-reversed calculation. While Figure $4 b$ clearly conveys the visual 
impression of diffusive transport, the individual trajectories are not resolvable.

Finally, we consider radiation belt-ions having $\mu=$ $30 \mathrm{MeV} / \mathrm{G}$. These would have energies of $335 \mathrm{keV}$ at $R=3$. The quiescent drift period $\left(\tau_{3}=44 \mathrm{~min}\right)$ of such ions on the shell that intersects the dusk meridian at $R=3$ (and found to have $L=3.00$ ) is less than $1 / 4$ of our model storm's duration. A plot of the quiescent adiabatic drift paths for $\mu=30 \mathrm{MeV} / \mathrm{G}$ (Figure 4c) shows no stagnation point earthward of the neutral line on the dawn meridian. When the storm is simulated, the time-reversed ion transport for $\mu=30$ $\mathrm{MeV} / \mathrm{G}$ again resembles radial diffusion (see Figure $4 d$ ). About half of the representative particles would have reached the final drift path from higher $L$ values and half from lower. More precisely, those particles that would have been on the dawn side at storm onset are the ones that have been transported inward since their initial gradient drift is toward the night side. Since the final steady-state drift paths lie well inside the region bounded by the stormtime separatrix, there is little chance of stormtime access from an open drift path.

We also ran simulations forward in time from $t=0$ for $\mu \gtrsim 15 \mathrm{MeV} / \mathrm{G}$ so as to follow the dispersal of ions from a common initial drift path and in order to estimate (see below) the corresponding radial-diffusion coefficient. Not surprisingly, the resulting stormtime transport was qualitatively similar to that shown in Figure 4, although the particle populations followed in the time-forward simulations were implicitly different from those followed in the time-reversed simulations. This type of transport was typical for ions having $\mu \gtrsim 13$ $\mathrm{MeV} / \mathrm{G}$, which corresponds to energies $\gtrsim 145 \mathrm{keV}$ at $R=3$.

Figures 3 and 4 thus illustrate a transition between modes of particle access to the stormtime ring current. Ring-current ions having $\mu \lesssim 3 \mathrm{MeV} / \mathrm{G}$ undergo direct convection from open (plasmasheet) drift paths to closed drift shells at $R \sim 3$, while radiation-belt ions having $\mu \gtrsim 30 \mathrm{MeV} / \mathrm{G}$ respond to the same impulsive enhancement of the convection electric field in a manner that resembles radial diffusion among closed drift shells [cf. Lyons and Schulz, 1989]. The transition between these two idealized modes of access occurs at $\mu \sim$ 5-15 MeV/G and thus (as might have been expected) for ring-current particles whose adiabatic drift periods are comparable to the duration of our model storm's main phase. These transitional particles have energies $\sim 55-165 \mathrm{keV}$ at $R=3$ and are thus quite representative [cf. Williams, 1981] of the ring current as a whole. Simplified analytical descriptions of the particle transport pertain most directly to these limiting cases (i.e., to $\mu \lesssim 3 \mathrm{MeV} / \mathrm{G}$ and to $\mu \gtrsim 30 \mathrm{MeV} / \mathrm{G}$, respectively), but application of the simplified analyses to intermediate $\mu$ values is similarly instructive for comparisons with our numerical results. Such comparisons are undertaken in the next two sections.

Although our simulation results are presented in this paper as pertaining to singly charged ions, the results can be made applicable to multiply charged ions by replacing $\mu$ in all our expressions with $\mu / Z$, where $Z$ is the ionic charge state. For example, our results for singly charged ions having $\mu=3 \mathrm{MeV} / \mathrm{G}$ (shown in the upper panels of Figure 3) would apply equally well to doubly charged ions having $\mu=6 \mathrm{MeV} / \mathrm{G}$.

\section{Direct Convective ACCesS}

Lyons and Williams [1980] introduced a simple model for the direct convection of low-energy particles from one $L$ value to another. In their model a particle is assumed to remain at fixed local time, while being transported radially by an uniformly enhanced azimuthal electric field. Their model implicitly entailed a uniform convection electric field but can be generalized for application to our model. By treating $\cos \phi$ and $\Delta V$ as constant in time, we could integrate (6) to obtain the condition

$$
\begin{array}{r}
\Delta V \Delta t=\frac{\mu_{E} L^{*}}{R_{E} \cos \phi}\left[\frac{1}{L_{f}^{2}}-\frac{1}{L_{i}^{2}}+\frac{2}{k}\left(\frac{1}{L_{i}}-\frac{1}{L_{f}}\right)\right. \\
\left.+\frac{2}{k^{2}} \ln \left(\frac{1+k / L_{f}}{1+k / L_{i}}\right)\right]
\end{array}
$$

for transport from $L_{i}$ to $L_{f}$ (interpreted here as the initial and final field-line labels). The parameter $k$ in (10) denotes $\left(\Delta V / V_{0}\right) L^{*}$. By taking the limit $k \rightarrow 0$, we recover from (10) the steady-state convection time

$$
\Delta t=\frac{2 \mu_{E}\left(L^{*}\right)^{2}}{3 V_{0} R_{E} \cos \phi}\left[\frac{1}{L_{f}^{3}}-\frac{1}{L_{i}^{3}}\right]
$$

that would have been deduced directly from (6) by setting $\Delta V(t) \equiv 0$. By taking the limit $k \rightarrow \infty\left(V_{0} \rightarrow 0\right)$, we recover from (10) the storm-associated convection time

$$
\Delta t=\frac{\mu_{E} L^{*}}{R_{E} \cos \phi \Delta V}\left[\frac{1}{L_{f}^{2}}-\frac{1}{L_{i}^{2}}\right]
$$

obtained by Lyons and Williams [1980]. These last two expressions remind us that (10) should be applied only over time intervals during which $\cos \phi$ is reasonably constant. Application of (11) to longer time intervals would falsely predict eventual access to arbitrarily small $L_{f}$ even for arbitrarily small $V_{0}$ and/or $\Delta V$, whereas corotation and/or gradient drift actually prevent this.

Lyons and Williams [1980] had envisioned direct convective access from closed trajectories. However, our simulation results (see right-hand panels of Figure 3) indicate that many low- $\mu$ particles reach $R=3$ along open-drift trajectories and, in particular from $L^{*}$ during our model storm. We want to compare the transport times encountered in our stormtime simulations with predictions based on the simple model of direct convection for these particles. Since $\Delta V$ varies with time in our model storm, we need to reinterpret $\Delta V(t) \Delta t$ in (10) as the time integral of $\Delta V(t)$. We assume that $\phi$ has been constant during most of the transport for low- $\mu$ particles and thus replace $\phi$ by $\phi^{*}$ in (10), where $\phi^{*}$ is the particle's local time when it crossed the neutral line $\left(L^{*}\right)$ at time $t=t^{*}$. We use the $180-\mathrm{kV}$ value found from $\langle\Delta V(t)\rangle$ during our model storm in order to treat the parameter $k \equiv\left(\Delta V / V_{0}\right) L^{*}$ as a constant on the right-hand side of (10). Thus, we obtain 


$$
\begin{aligned}
I\left(t^{*}\right) \equiv \int_{t^{*}}^{\infty} \Delta V(t) d t \\
=\frac{\mu_{E} L^{*}}{R_{E} \cos \phi^{*}}\left[\frac{1}{L_{f}^{2}}-\frac{1}{L^{* 2}}+\frac{2}{k}\left(\frac{1}{L^{*}}-\frac{1}{L_{f}}\right)\right. \\
\\
\left.\qquad \frac{2}{k^{2}} \ln \left(\frac{1+k / L_{f}}{1+k / L^{*}}\right)\right]
\end{aligned}
$$

as our generalization of (10). We plot the right-hand side of equation (12) against $\phi^{*}$ as the solid curve in Figure 5. This curve represents our generalization of the convection-time estimate made by Lyons and Williams [1980].

For comparison, we compute and plot $I\left(t^{*}\right)$ for each representative particle by integrating $\Delta V(t)$, as given by (4), over the time during which the particle was being transported from the neutral line to the final drift path of interest. We found that 7 of the 12 representative ions having $\mu=3 \mathrm{MeV} / \mathrm{G}$ were transported to $L=3.14$ in this manner. The corresponding values of $I\left(t^{*}\right)$ are plotted as solid circles in Figure 5. Agreement with the direct-convective idealization is good for those ions which required the least $I\left(t^{*}\right)$, around $200-300 \mathrm{kV}$ $\mathrm{hr}$, to get to $L_{f}$. Deviations from (12) are large for those ions which required larger values of $I\left(t^{*}\right)$, presumably because those ions have taken a more circuitous route to the final drift shell of interest.

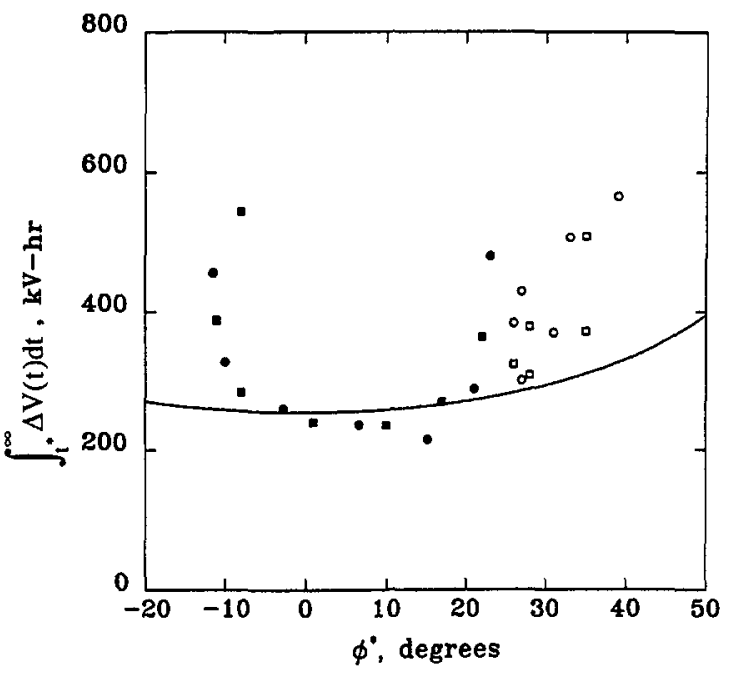

Fig. 5. The time-integral $I\left(t^{*}\right) \equiv \int_{0}^{\infty} \Delta V(t) d t$ required for convective transport of a particle from the neutral line $\left(L^{*}\right)$ to the final drift shell of interest, given by the right-hand side of (12), for fixed magnetic local time $\phi=\phi^{*}$, is plotted as a solid curve. Solid and open circles represent values of $I\left(t^{*}\right)$ obtained from simulated trajectories of representative ions having $3 \mathrm{MeV} / \mathrm{G}$ and $10 \mathrm{MeV} / / \mathrm{G}$, respectively, under our model storm (cf. Figure $2 b$ ). Solid and open squares represent values of $I\left(t^{*}\right)$ obtained from simulated trajectories of representative ions having $3 \mathrm{MeV} / \mathrm{G}$ and $10 \mathrm{MeV} / \mathrm{G}$, respectively, under the idealized "boxcar" storm model specified by (21) with $\Delta V=200 \mathrm{kV}$ over a 3 -hr main phase.
As an additional test of (12) we make use of an earlier simulation we had run for ions having $\mu=3 \mathrm{MeV} / \mathrm{G}$ in a storm model wherein $\Delta V(t)$ was represented as a boxcar function of time (i.e., held constant at $\Delta V=200$ $\mathrm{kV}$ over a 3-hr main phase). Values of the corresponding $I\left(t^{*}\right) \equiv \Delta V \Delta t=\left(3 \mathrm{hr}-t^{*}\right) \times 200 \mathrm{kV}$ for those 7 of the 12 representative ions that were transported from the neutral line to the final drift path of interest during this idealized storm are plotted as solid boxes in Figure 5. Agreement with (12) is best for those ions which had the shortest transport times $(\sim 1.2 \mathrm{hr})$ and thus the smallest values $(\sim 240 \mathrm{kV}-\mathrm{hr})$ for $I\left(t^{*}\right)$. We have omitted plotting $I\left(t^{*}\right)$ in Figure 5 for the one representative ion having $\mu=3 \mathrm{MeV} / \mathrm{G}$ that was on an open trajectory but located at $L<L^{*}$ at storm onset (see Figure $36)$. This is because the right-hand side of $(10)$ really applies with approximately constant $k$ only to particles for which $L_{i}=L^{*}$, since $k=0$ essentially by definition for $t<0$. We expect that $I\left(t^{*}\right)$ for this particular representative ion would not agree well with (12) in any case, since its circuitous path leads to $I\left(t^{*}\right) \approx 600 \mathrm{kV}-\mathrm{hr}$.

We make similar comparisons in Figure 5 for ions having $\mu=10 \mathrm{MeV} / \mathrm{G}$. We plot values of $I\left(t^{*}\right)$ as open circles for those 6 of the 12 representative ions that were transported from the neutral line to the final drift path of interest during our applied model storm (see Figure $2 b$ ). Likewise, we plot values of $I\left(t^{*}\right)=\left(3 \mathrm{hr}-t^{*}\right) \times 200$ $\mathrm{kV}$ as open boxes for those 5 of the 12 representative ions that were transported from the neutral line to the final drift path of interest during our boxcar idealization of a model storm. Again, agreement with (12) is good only for ions having short transport times $(t \sim 1.5 \mathrm{hr})$ in the boxcar idealization and relatively small values $(\lesssim 300 \mathrm{kV}-\mathrm{hr})$ for $I\left(t^{*}\right)$ in general. However, a smaller fraction of the $\mu=10 \mathrm{MeV} / \mathrm{G}$ ions than of the $\mu=3$ $\mathrm{MeV} / \mathrm{G}$ ions agree well with (12), probably because the effect of gradient- $B$ drift on the transport is greater for $\mu=10 \mathrm{MeV} / \mathrm{G}$ than for $\mu=3 \mathrm{MeV} / \mathrm{G}$.

In view of the good agreement between values of $I\left(t^{*}\right)$ shown by small circles and by corresponding boxes in Figure 5, we infer that (12) will provide a good estimate for the minimum access time (for particles transported most directly inward by mean stormtime $\Delta V$ ) also in this situation. As in the case in Figure 5, however, we expect to find that (12) will not provide a very good access-time estimate for particles that have taken a more circuitous route to the drift path of interest.

\section{DIFFUSION AND QUASI-DIFFUSION COEFFICIENTS}

In contrast to the lower- $\mu$ ions, the transport of highenergy ions with $\mu \gtrsim 15 \mathrm{MeV} / \mathrm{G}$ (cf. Figure 4) resembles radial diffusion. In this section, we make detailed quantitative comparisons of radial diffusion coefficients obtained from our simulations with quasilinear theory [Fälthammar, 1965] to show that the transport is indeed well described as radial diffusion. For cases in which all 12 representative ions are found to have been transported from initially'closed drift shells to the final closed drift shell which intersects the dusk meridian at $R=3$, we have computed the $L$ values $L_{i}$ (and $L_{f}$ ) of the respective initial (and final) drift shells in accordance with (9) and have constructed the quantity 


$$
\begin{gathered}
D_{L L}^{\text {sim }} \equiv\left(\frac{1}{2 T}\right)\left(\frac{d L_{f}}{d L_{f}^{-1}}\right)^{2}\left[\left\langle\left(L_{i}^{-1}-L_{f}^{-1}\right)^{2}\right\rangle\right. \\
\left.-\left\langle\left(L_{i}^{-1}-L_{f}^{-1}\right)\right\rangle^{2}\right] \\
=\left(\frac{L^{4}}{24 T}\right)\left[\sum_{i=1}^{12}\left(L_{i}^{-1}-L_{f}^{-1}\right)^{2}-\left[\sum_{i=1}^{12}\left(L_{i}^{-1}-L_{f}^{-1}\right)\right]^{2}\right],
\end{gathered}
$$

where $T(=3 \mathrm{hr}$ ) denotes the "duration" of the main phase of the model storm. The transport coefficient $D_{L L}$ defined by (13) corresponds to the familiar diffusion coefficient obtained in quasilinear theory [Fälthammar, 1965], which applies in the limit $\max _{i}\left|L_{i}^{-1}-L_{f}^{-1}\right| \rightarrow 0$. The quantity $D_{L L}^{\text {sim }}$ is thus a measure of the variance among the initial third adiabatic invariants of particles situated on the final drift shell of interest. We also computed diffusion coefficients $D_{L L}^{\text {sim }}$ from simulations run forward in time by interchanging the indices $i$ and $f$ in (13). The diffusion coefficients $D_{L L}^{\text {sim }}$ obtained from simulations run forward and backward in time are not very different, $80 \%$ of them being within a factor of 1.5 of each other, although they pertain implicitly to different sets of particles.

The resonant-particle formulation [Fälthammar, 1965; Schulz and Lanzerotti, 1974, pp. 81-95] of radialdiffusion theory leads to a diffusion coefficient of the form

$$
D_{L L}=\frac{L^{6} R_{E}^{4}}{4 \mu_{E}^{2}} \hat{E}\left(\frac{\Omega_{3}}{2 \pi}\right)
$$

where $\hat{E}(\omega / 2 \pi)$ is the spectral-density of the (quasiuniform) equatorial electric field in the inner magnetosphere and $\Omega_{3} / 2 \pi$ is the particles' drift frequency. Since the strength $\Delta E(t)$ of the fluctuating part of $-\nabla \Phi_{E}$ is given, following (3), by

$$
\Delta E(t)=-\left(\frac{1}{2 L^{*} R_{E}}\right) \Delta V(t)
$$

in the equatorial plane at $R \ll L^{*}$, the spectral density $\hat{E}\left(\Omega_{3} / 2 \pi\right)$ could alternatively be written as $\left(1 / 2 L^{*}\right.$ $\left.R_{E}\right)^{2} \hat{V}\left(\Omega_{3} / 2 \pi\right)$, where $\hat{V}(\omega / 2 \pi)$ denotes the spectraldensity function of $\Delta V(t)$, which is the storm-associated enhancement in the cross-tail potential drop. The model for $\Delta V(t)$ specified by (4) leads to a spectraldensity function of the form

$\hat{E}(\omega / 2 \pi)=\frac{1}{T} \frac{\tau^{2}}{1+\omega^{2} \tau^{2}} \sum_{i=1}^{N} \sum_{j=1}^{N} \Delta E_{i} \Delta E_{j} \cos \left[\omega\left(t_{j}-t_{i}\right)\right]$

where the summations extend over all $N$ impulses (see the Appendix for details of the derivation). The corresponding diffusion coefficient given by quasilinear theory is

$$
D_{L L}^{q l}=\frac{\tau^{2} L^{6} R_{E}^{2}}{16 T \mu_{E}^{2}\left(L^{*}\right)^{2}} \sum_{i=1}^{N} \sum_{j=1}^{N} \frac{\Delta V_{i} \Delta V_{j} \cos \left[\Omega_{3}\left(t_{j}-t_{i}\right)\right]}{1+\Omega_{3}^{2} \tau^{2}} .
$$

The impulse-onset times $t_{i}$ and $t_{j}$ in our model storm are not quite randomly spaced in time because of our dead time constraint. A radial-diffusion model in which an infinite number of impulses (with an amplitude distribution symmetric about zero) occur purely at random would lead to a statistical cancellation of the cross terms $(j \neq i)$ in (17). In this case, one would recover a radial-diffusion coefficient of the form found by Cornwall [1968] and quoted by Lyons and Schulz [1989]:

$$
\tilde{D}_{L L}^{q l}=\frac{\tau^{2} L^{6} R_{E}^{2}}{16 T \mu_{E}^{2}\left(L^{*}\right)^{2}\left(1+\Omega_{3}^{2} \tau^{2}\right)} \sum_{i=1}^{N}\left(\Delta V_{i}\right)^{2} .
$$

The spectral density function $\hat{E}(\omega / 2 \pi)$ corresponding to our model storm (see Figure $2 b$ ) is plotted as the solid curve in Figure 6. Its most notable feature is an extreme lack of smoothness [cf. Lanzerotti and Wolfe, 1980]. The spectrum is so variable because the impulse onsets $(i)$ in any individual storm modeled by (4) occur at specific times. The dashed curve in Figure 6 represents the "smoothed" spectrum obtained from (16) by neglecting the cross terms $(j \neq i)$ for the same model storm. We have introduced the smoothed spectrum in order to provide a standard of reference, equivalent to that introduced by Cornwall [1968], against which to compare our results for $D_{L L}^{\text {sim }}$ and $D_{L L}^{d l}$ (especially when these differ significantly from each other).

Since $\hat{E}(\omega / 2 \pi)$ does not vary smoothly with $\omega$, the quasilinear diffusion coefficient $D_{L L}^{q l}$ is not a smooth function of $\mu$. The solid curve in Figure 7 represents the variation of $D_{L L}^{q l}$ with $\mu$ for ions on drift shells which intersect the dusk meridian at $R=3$. In order to plot the quasilinear diffusion coefficient as a continuous function of $\mu$, we used the empirical formulas

$$
\tau_{3}=\frac{\left[1+(0.17 / \mu)+\left(0.61 / \mu^{2}\right)\right] \times 10^{4}}{7.9434 \mu-6.9418} \min
$$

and

$$
L=\left[0.335469-(0.03 / \mu)-0.11 \mu^{-5 / 2}\right]^{-1},
$$

which we inferred from our steady-state numerical results by setting $\Delta V(t)=0$ in (6) and (7). Diffusion coefficients obtained from simulations run forward (solid circles) and backward (open circles) in time are also plotted in Figure 7 for comparison. Agreement of the diffusion coefficients obtained from the simulations with quasilinear theory is surprisingly good despite the strong variability of $D_{L L}^{q l}$ with $\mu$. We find typically (i.e, in $>80 \%$ of the available cases) that $D_{L L}^{\mathrm{sim}}$ and $D_{L L}^{q l}$ agree within a factor of 4 . Quasilinear theory even accounts for the $\mu$ values (e.g., $\mu=75 \mathrm{MeV} / \mathrm{G}$ and $\mu$ $=80 \mathrm{MeV} / \mathrm{G}$ ) at which the diffusion coefficients computed from the simulation are especially small. However, there are still some discrepancies between $D_{L L}^{\text {sim }}$ and $D_{L L}^{q l}$, typically at $\mu$ values corresponding to sharp "peaks" or "valleys" in the electric spectral density. Cases in which the quasilinear diffusion coefficient underestimates $D_{L L}^{\text {sim }}$ (e.g., for $\mu=25$ and $80 \mathrm{MeV} / \mathrm{G}$ ) typically correspond to "valleys" in the spectral density. Conversely, cases in which the quasilinear diffusion coefficient $D_{\mathrm{L} L}^{q l}$ overestimates $D_{L L}^{\mathrm{sim}}$ (e.g., for $\mu=$ $30,60,90,100,120$, and $150 \mathrm{MeV} / \mathrm{G}$ ) typically corre- 


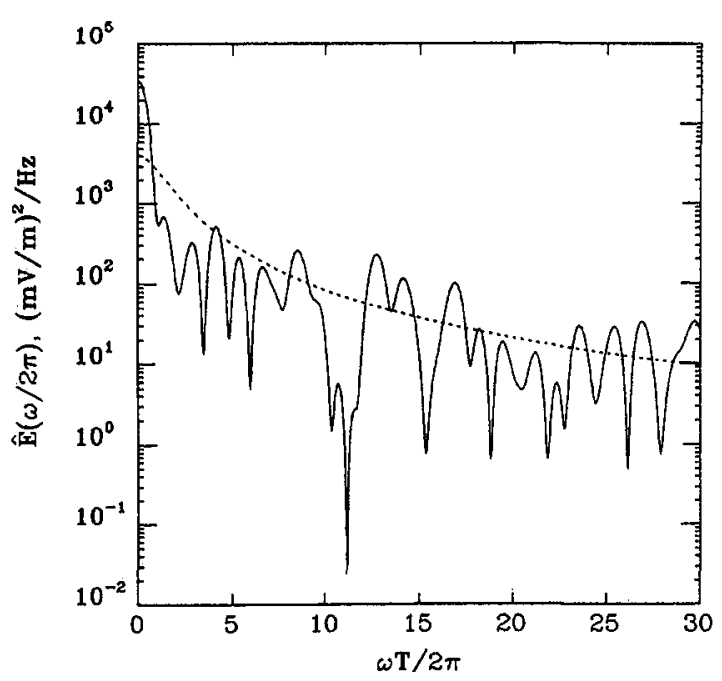

Fig. 6. The spectral density of the electric-field fluctuations in our $(T=3 \mathrm{hr})$. The dashed curve represents the "smoothed" spectral density computed as if the same impulses had been uncorrelated with respect to onset time $\left(t_{i}\right)$ and sign of amplitude $\left(\Delta V_{i}\right)$. The dashed curve corresponds implicitly to the electric-impulse model considered by Cornwall [1968].

spond to "peaks" in the electric spectral density. For these cases, we find that the smoothed quasilinear diffusion coefficient $\tilde{D}_{L L}^{q l}$ (dashed curve in Figure 7) given by (18) generally agrees better with $D_{L L}^{\text {sim }}$ than does the unsmoothed quasilinear diffusion coefficient $D_{L L}^{q l}$. In view of the extreme lack of smoothness in the spectral density this tendency of $\tilde{D}_{L}^{q l}$ to reduce the discrepancies between $D_{L L}^{\text {sim }}$ and $D_{L L}^{4 t}$ led us to suspect that an appeal to resonance-broadening might improve upon our quasilinear results for $D_{L L}^{q l}$. The idea was that model storm is plotted (solid curve) against normalized frequency

a spread in drift frequencies would accumulate among the 12 representative particles as a consequence of their spread in $L$. However, we found that such resonancebroadening only slightly smoothed the sharp minima and maxima in $D_{L L}^{q l}$ so that the resulting diffusion coefficients $D_{L \dot{L}}^{r b}(\mu)$ still differed from $D_{L L}^{\mathrm{sim}}$ by a factor $\gtrsim 3$ in about $20 \%$ of the cases [Chen et al., 1992] instead of by a factor of $\gtrsim 4$ (as found here).

Figures $8 a$ and $8 b$ show comparisons of the diffusion coefficients for model storms identical to that shown in

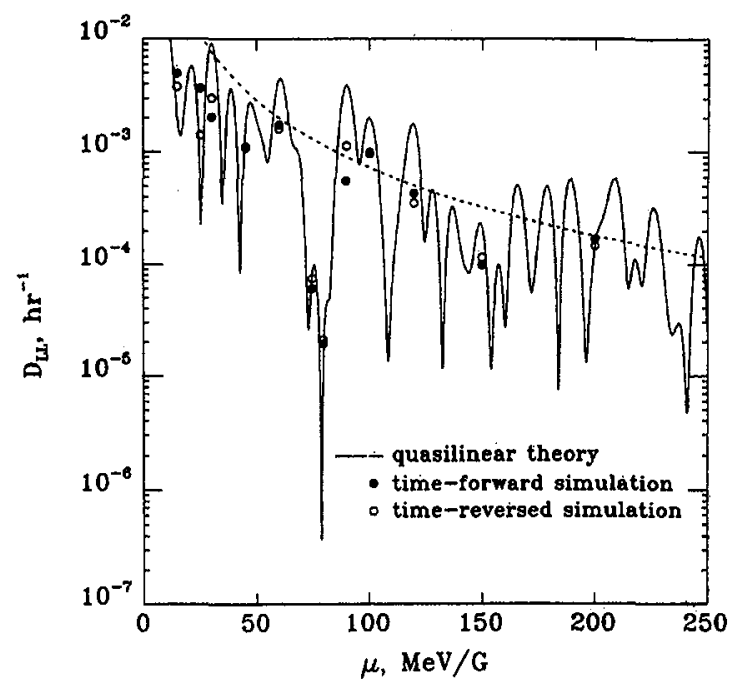

Fig. 7. Solid and open circles represent values of radial-diffusion coefficient $D_{L L}^{\text {sim }}$ obtained via (13) from time-forward and timereversed simulations, respectively. Quasilinear diffusion coefficient $D_{L L}^{q l}$ (solid curve) is given by (17) and its "smoothed" counterpart $\tilde{D}_{L L}^{4 L}$ (dashed curve) by (18) for model storm defined by Figure

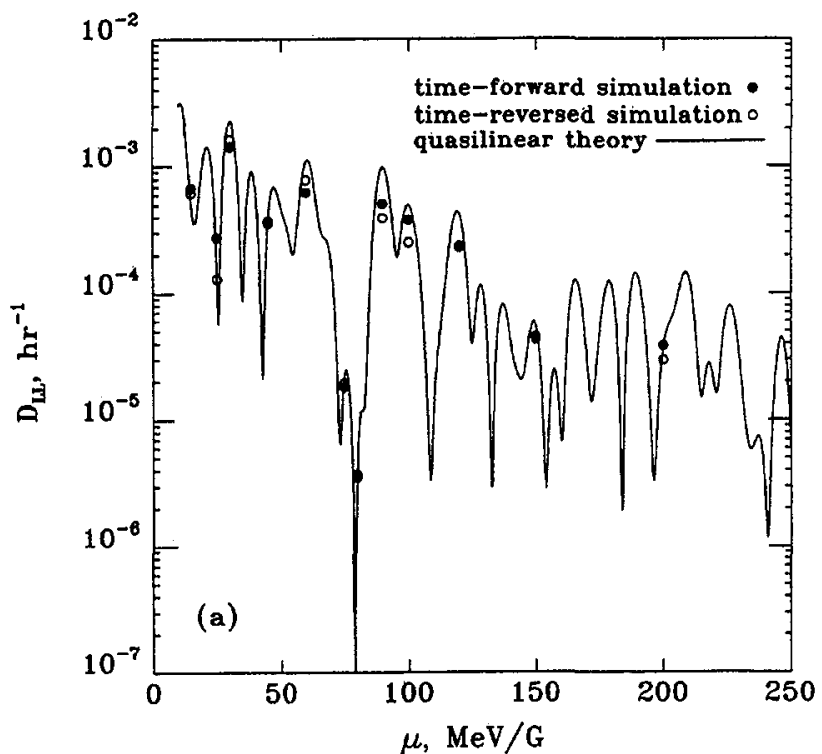

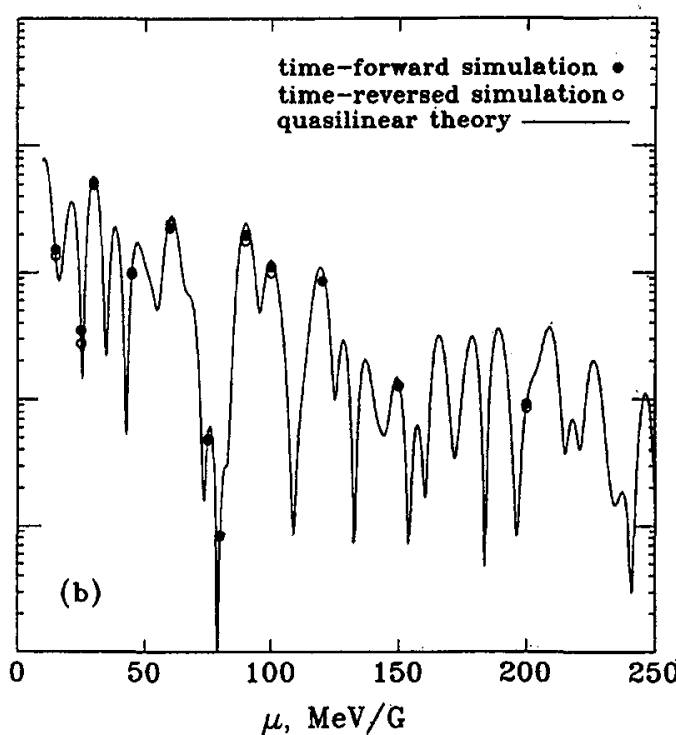

Fig. 8. Solid and open circles represent values of radial-diffusion coefficient $D^{\text {sim }}$ obtained via (13) from timeforward and time-reversed simulations, respectively. Quasilinear diffusion coefficient $D_{L L}^{q l}$ (solid curve) is given by (17) as an implicit function of $\mu$ for model storms equivalent to that defined by Figure $2 b$ except that the impulse amplitudes in $\Delta V(t)$ have been reduced by a factor of 2 (hence $D_{L L}^{q l}$ by a factor of 4 ) in Figure $8 a$ and by a factor of 4 (hence $D_{L L}^{q l}$ by a factor of 16 ) in Figure $8 b$. 
Figure $2 b$, except that the impulse amplitudes $\Delta V_{i}$ were reduced by factors of 2 and 4, respectively. Agreement

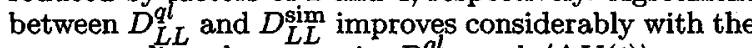
corresponding decreases in $D_{L L}^{q l}$ and $\langle\Delta V(t)\rangle$, as we might have expected. The discrepancies between these estimates of the diffusion coefficients amount typically (i.e., in $>80 \%$ of cases available for comparison) to a factor of $\lesssim 1.3$ and $\lesssim 1.2$ when $\Delta V(t)$ is reduced by factors of 2 and 4 , respectively. This agreement is remarkably good in view of the fact that the quasilinear diffusion coefficient $D_{L L}^{q l}$ varies irregularly through 4 orders of magnitude for $15 \mathrm{MeV} / \mathrm{G} \lesssim \mu \lesssim 250 \mathrm{MeV} / \mathrm{G}$. Moreover, the agreement between diffusion coefficients obtained from simulations run forward (solid circles) and backward (open circles) in time improves notably as the impulse amplitudes in $\Delta V(t)$ are reduced. We find in $>80 \%$ of the cases examined that the discrepancy between "time-forward" and "time-reversed" estimates for $D_{L L}^{\mathrm{sim}}$ amount to factors $\lesssim 1.5, \lesssim 1.3$, and $\lesssim 1.15$ for $\langle\Delta V(t)\rangle=180,90$, and $45 \mathrm{kV}$, respectively: Improved agreement with decreasing $\langle\Delta V(t)\rangle$ is not surprising since the quasilinear diffusion coefficient is formally invariant under time reversal. Quasilinear theory is supposed to approximate the simulated transport better and better as $\Delta V(t) \rightarrow 0$, this being the limit in which the ion populations represented in simulations run forward and backward in time become less and less distinct.

The surprisingly good agreement between $D_{L L}^{q l}$ and $D_{L L}^{\text {sim }}$ which we found for a storm modeled as a quasirandom sequence of impulsive enhancements in the convection electric field has led us to ask whether an even simpler storm model might lend itself to a successful quasi-linear description. As is noted above, we had successfully tested a boxcar representation,

$$
\Delta V(t)=\theta(t) \theta(T-t) \Delta V
$$

with $T=3 \mathrm{hr}$, for the description of direct convective access for $\mu \lesssim 13 \mathrm{MeV} / \mathrm{G}$. We thought it might be instructive also to compare the diffusion coefficients $D L L$ and $D_{L L}^{q l}$ from the application of (21) with each other and with the diffusion coefficients plotted for $\mu \gtrsim 15$ $\mathrm{MeV} / \mathrm{G}$ in Figure 7. Thus, we have set $\Delta V=200 \mathrm{kV}$ in (21) for the purpose of making these comparisons. We might expect a much poorer agreement between the diffusion coefficients $D_{L L}^{\text {sim }}$ and $D_{L L}^{q l}$ in this latter model for $\Delta V(t)$, but a formal substitution of (4) into (A2) via (16) nevertheless yields (see Appendix)

$$
\hat{E}(\omega / 2 \pi)=\frac{4(\Delta E)^{2}}{T} \frac{\sin ^{2}(\omega T / 2)}{\omega^{2}} .
$$

Substitution of this spectral-density function into (14) yields a diffusion coefficient of the form

$$
D_{L L}^{q l}=\frac{L^{6} R_{E}^{2}(\Delta V)^{2} T}{16 \mu_{E}^{2}\left(L^{*}\right)^{2}} \frac{\sin ^{2}\left(\pi T / \tau_{3}\right)}{\left(\pi T / \tau_{3}\right)^{2}}
$$

upon evaluation of the spectral density at the particle's quiescent drift frequency $\Omega_{3} / 2 \pi \equiv \tau_{3}^{-1}$. The quasilinear diffusion coefficient is plotted as the solid curve in Figure 9. Because of the sinusoidal factor in the spectral-density function, the quasilinear diffusion coefficient vanishes for $\tau_{3}=T /(n+1)$, with $n=0,1,2, \ldots$. Thus, particles nominally executing an integral number of drift periods during the storm would experience no net diffusion, having been transported as much inward as outward during their $n+1$ complete drift periods. For these cases the diffusion coefficient given by (23) will surely underestimate the actual transport coefficient, since in reality there must be a spread in the stormtime drift frequencies of the representative particles. The actual diffusion coefficient in sych cases could possibly be estimated by averaging $D_{L L}^{q l}$ over an appropriate bandwidth of drift frequencies [cf. Dungey, $1965 ;$ Schulz, 1975]. Here we smooth $D_{L L}^{q l}$ (perhaps excessively) by replacing the factor $\sin ^{2}\left(\pi T / \tau_{3}\right)$ with its mean value $(1 / 2)$ so as to obtain

$$
\tilde{D}_{L L}^{q l} \approx \frac{L^{6} R_{E}(\Delta V)^{2} \tau_{3}^{2}}{32 \mu_{E}^{2}\left(L^{*}\right)^{2} \pi^{2} T}
$$

for $\tau_{3} \ll \sqrt{2} \pi T \approx 800 \mathrm{~min}$ (this last inequality being well satisfied for the range of $\mu$ values considered in this study). The dashed curve in Figure 9 represents $\tilde{D}_{L L}^{q l}$. Since the boxcar function in $\Delta V(t)$ specified by. (21) is symmetric in time about $t=1.5 \mathrm{hr}$, the diffusion coefficients $D_{L L}^{\text {sim }}$ obtained from simulations run forward or backward in time are equal. We compare diffusion coefficients obtained from the simulation (solid circles) with the quasilinear diffusion coefficient (solid curve) and with the smoothed quasilinear diffusion coefficient (dashed curve) in Figure 9. We find, quite remarkably, that $\tilde{D}_{L L}^{q l}$ actually provides a good estimate (within a factor of $\sim 2$ ) for $D_{L L}^{\text {sim }}$ over the entire range of $\mu$ values for which the uniform enhancement in the convection

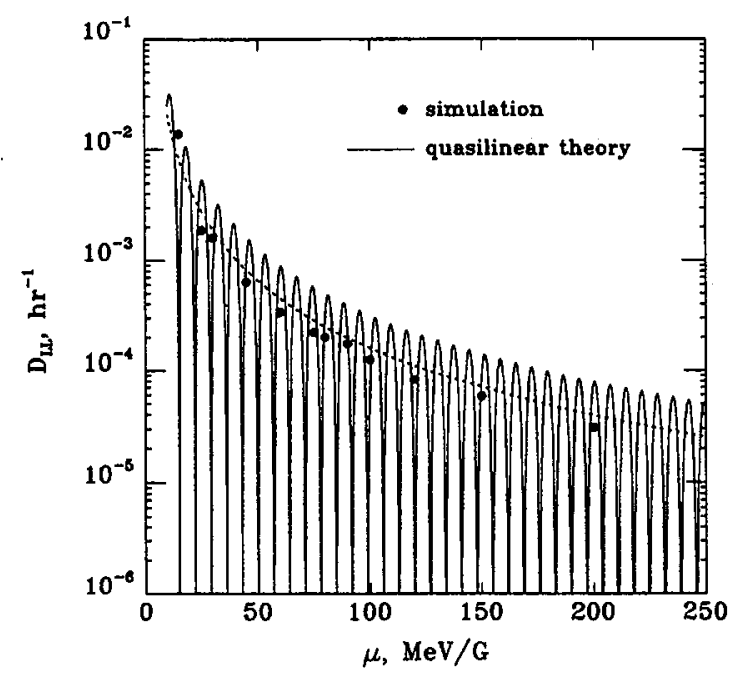

Fig. 9. Solid circles represent values of radial-diffusion coefficient $D_{L L}^{\text {sim }}$ obtained via (13) from guiding-center simulations (either time-forward or time-reversed). Quasilinear diffusion coefficient $\tilde{D}_{L L}^{4_{L}^{L}}$ (dashed curve) by (24) for idealized "boxcar" storm model defined by (21) with $\Delta V=200 \mathrm{kV}$ and $T=3 \mathrm{hr}$ (main phase duration). 
electric field transports particles entirely among closed drift shells. :We find also (not surprisingly) that the values of $D_{L L}^{\text {sim }}$. for ions at the larger $\mu$ values $(\mu \gtrsim 90$ $\mathrm{MeV} / \mathrm{G}$ ) is generally greater during an impulsive model storm based on (4) than for a boxcar model based on (21). However, a comparison between Figures 7 and 9 (note the one-decade scale offset) shows little systematic variation between values of $D_{L L}^{\text {sim }}$ deduced from (4) and from (21) for $\mu \lesssim 50 \mathrm{MeV} / \mathrm{G}$.

\section{SUMMARY AND CONCLUSIONS}

We have used a dynamical guiding-center simulation model to study the stormtime ion transport which leads to formation of the ring current and diffusion in the radiation belts. We have traced representative ions' guiding-center motion in response to model storm-associated impulses in the convection electric field for a range of ion $\mu$ values. Although our simulation results are presented in this paper as pertaining to singly charged ions, the results can be made applicable to multiply charged ions by replacing $\mu$ in all our expressions with $\mu / Z$, where $Z$ is the ionic charge state. By comparing our numerical results with previously formulated limiting idealizations of particle transport (convective access and radial diffusion), we have assessed the limits of validity of these approximations and have encountered several interesting nuances. A summary of our results follows:

1. For ions having drift periods that exceed the duration of the main phase of the storm $(\mu \lesssim 10 \mathrm{MeV} / \mathrm{G}$, hence $E \lesssim 110 \mathrm{keV}$ at $L \approx 3$ ), their inward transport to form the stormtime ring current is appropriately described as direct convective access [Lyons and Williams, 1980].

2. For ions having drift periods comparable to the duration of the main phase of the storm ( $\mu \sim 10-25$ $\mathrm{MeV} / \mathrm{G}$, hence $110 \mathrm{keV} \lesssim E \lesssim 280 \mathrm{keV}$ at $L \approx 3$ ), there is a transition between direct convective access and transport that resembles radial diffusion.

3. The mean "stormtime" separatrix constitutes an idealized boundary between closed and open particle trajectories for a given $\mu$. The location of the stormtime separatrix relative to the final (closed) adiabatic drift path of interest has implications concerning the type of particle transport encountered. For example, any portion of the final drift shell of interest that lies outside the stormtime separatrix is accessible to particles from previously open drift paths. Conversely, if the final drift shell of interest lies entirely earthward of the stormtime separatrix, then particles must be transported from closed drift paths.

4. For ions having drift periods considerably shorter than both $T$ (the duration of the main phase of the storm), the transport is well described by radial diffusion.

5. The electric spectral density of our model storm is extremely variable and leads to a highly irregular variation of radial-diffusion coefficients $D_{L L}^{\text {sim }}$ obtained from our simulations with $\mu$. The variation is similar to that found from observational data in spectral densities extracted by Lanzerotti and Wolfe [1980] and thus may be realistic.
6. Despite their high variability with $\mu$, the diffusion coefficients $D_{L L}^{s i m}$ obtained from the simulations agree surprisingly well with the quasilinear estimates [cf. Fälthammar, 1965] for $D_{L L}$ at $L \approx 3$ for higher$\mu$ ions $(\mu \gtrsim 15 \mathrm{MeV} / \mathrm{G}$, hence $E \gtrsim 165 \mathrm{kV}$ at $L \approx 3$ ). Agreement between $D_{L L}^{\text {sim }}$ and the quasilinear diffusion coefficient $\left(D_{L L}^{q l}\right)$ improves when the impulse amplitudes in the convection electric field are decreased.

An important implication of our study is that lowerenergy ( $E \lesssim 110 \mathrm{keV}$ ) ring-current ions at $L \approx 3$ are freshly injected there from open adiabatic trajectories, whereas the higher-energy ( $E \gtrsim 150 \mathrm{keV}$ ) ring-current population consists of a mixture of freshly injected and previously trapped ions (both having been transported quasi-diffusively to the drift shell of interest). Composition measurements on the ring-current particle population region before and after the main phase of a geomagnetic storm might provide a test of this interpretation.

\section{APPENDIX}

\section{CONSTRUCTION AND ANALYSIS OF MODEL STORM}

In order to determine a reasonable set of $t_{i}$ for use in (4) to construct our model storm, we have generated 1800 random real numbers between 0 and 100 and have sorted these into 100 bins by noting the integer part of each random number. Although our procedure strictly (rather than just probabilistically, as it should have) constrains the total to 1800 chosen random numbers per 100 bins and thus the mean to 18 chosen random numbers per bin, we have found that the distribution of random numbers among bins is well approximated by a Poisson distribution with a mean of 18 per bin. In other words, the number of bins containing $N$ chosen random numbers is well approximated by $100\left(\bar{N}^{N} / N !\right) \exp (-\bar{N})$ with $\bar{N}=18$. A chi-square test of our distribution of random numbers against a Poisson distribution with $\bar{N}=18$ yields $\chi^{2}=9.50$ for 14 degrees of freedom. The probability $P$ that any alternative distribution of random numbers would have yielded a smaller deviation from the expected bin values for 14 degrees of freedom is $P\left(\chi^{2} \leq 9.50\right)=0.20$ [see Hogg and Craig, 1959, p. 237]. Since this $P$ lies well between 0.1 and 0.9 , the $\chi^{2}$ test can be interpreted as having confirmed a Poisson distribution with $\bar{N}=18$ [cf. Evans, 1955, p. 775].

Each bin represents a 3-hr interval from the ensemble of model storms. Our intent is that, on average, 9 actual impulses (identified by the $t_{i}$ ) should occur during the "typical" main phase, in which case half of the "typical" 3-hr interval would be disqualified by "dead time" from containing a $t_{i}$. We multiply the fractional part of each chosen random number by $180 \mathrm{~min}$ and identify the earliest time thus generated in each storm of the ensemble with $i=1$ in (4). Since we impose a 10-min "dead time" after each $t_{i}$ in (4), we reject subsequent random times that exceed any such $t_{i}$ by less than $10 \mathrm{~min}$. In other words, $i=2$ in (4) corresponds to the next random number that exceeds the smallest random number in the same bin by at least $1 / 18$, and so on. Thus, the distribution of actual impulse start times $t_{i}$ is significantly distorted relative to a Poisson distribution with $\bar{N}=9$ [cf. Müller, 1973, 1974], even 
though there are 9 impulses in the "typical" 3 -hr main phase or 3 substorms/hr on average. For example, a Poisson distribution with $\bar{N}=9$ would have yielded 38 of 100 storms with $9 \pm 1$ impulses, whereas we find that 77 of our 100 randomly generated main phases contain $9 \pm 1$ "actual" impulses. Indeed, our distribution corresponds more closely to a Müller distribution, based on a Poisson distribution with $\bar{N}=18$ but distorted by a 10-min "dead time" [cf. Müller, 1973, 1974], according to which we should have expected 67 of 100 storms to contain $9 \pm 1$ "actual" impulses. A chi-square test of our distribution against a Müller distribution yields $\chi^{2}=4.13$ for 5 degrees of freedom. The probability $P$ that any alternative distribution of random numbers would have yielded a smaller value of $\chi^{2}$ for 5 degrees of freedom is $P\left(\chi^{2} \leq 4.13\right)=0.47$, and so the $\chi^{2}$ test confirms [cf. Evans, 1955, p. 775] that our ensemble of model storms satisfies the appropriate Müller distribution. Since our dead time constraint disqualifies randomly chosen candidate start times that follow too closely upon actual impulses, we find that the spacing between actual impulses tends to be more nearly even than random.

We have constructed our ensemble of model storms to have (on average) 9 impulses with 20-min decay times during a 3-hr main phase, we should have expected the time integral of $\Delta V(t)$ from $t_{1}$ to $\infty$ to be $\sim 600 \mathrm{kV}$ $\mathrm{hr}$. Indeed, the time integral of $\Delta V(t)$ from $t_{1}$ to $\infty$ for our prototypical storm is $594 \mathrm{kV}$-hr. We have noted above that $\langle\Delta V(t)\rangle$ for our model storm is $180 \mathrm{kV}$. The ensemble-averaged $\langle\Delta V(t)\rangle$ among the 100 storms that we constructed turned out to be $184 \mathrm{kV}$. This means that the time integral of $\Delta V(t)$ from $t_{1}$ to $t_{1}+3 \mathrm{hr}$ would have been $540 \mathrm{kV}-\mathrm{hr}$ and $552 \mathrm{kV}-\mathrm{hr}$ for our model storm and the ensemble, respectively, but this 3 -hr interval of integration excludes a significant portion of the final impulse.

Quasilinear radial-diffusion theory [Fälthammar, 1965] requires us to obtain the spectral density function of the electric field associated with the realization of our model storm that is illustrated in Figure $2 b$. Blackman and Tukey $[1958$, p. 7] define the spectral density function $\hat{E}(\omega / 2 \pi)$ as follows:

$$
\hat{E}(\omega / 2 \pi) \equiv \lim _{T \rightarrow \infty} \frac{1}{T}\left|\int_{-T / 2}^{T / 2} \Delta E(t) \exp (-i \omega t) d t\right|^{2}
$$

for $-\infty<\omega<\infty$. We need to modify this definition for use in (4) because we regard the diffusion coefficient as a function of time and presume it to be negligible for $t<0$ and for $t>3 \mathrm{hr}$. However, we would not wish to truncate the integral in (A1) at time $t=3 \mathrm{hr}$, since the contribution of the last few impulses to particle transport is not negligible until somewhat later. We truncate our simulations of particle transport during our model storm around $t=6 \mathrm{hr}$ or later, but that choice is quite arbitrary.

There would be no dilemma concerning the appropriate definition of $\hat{E}(\omega / 2 \pi)$ if the dynamical process being modeled were statistically stationary [cf. Blackman and Tukey, 1958, pp. 4-7], but in that case we would not be modeling a storm. We adopt the definition

$$
\hat{E}(\omega / 2 \pi) \equiv \frac{1}{T}\left|\int_{-\infty}^{\infty} \Delta E(t) \exp (-i \omega t) d t\right|^{2},
$$

with $T(=3 \mathrm{hr}$ ) being the span of time over which impulse onsets can occur, as the compromise appropriate to the present scenario.

The quantity actually simulated in the analysis prescribed by (13) is not the instantaneous $D_{L L}$ but rather its time integral, which corresponds to the ensemble of ion transitions between initial and final $L$ values. We have identified this time integral with $T D_{L L}^{\text {sim }}$. According to the classical analogue of Heisenberg's uncertainty principle, it would not be meaningful to specify $\hat{E}(\omega / 2 \pi)$ as a continuous function of both frequency and time. In effect, however, a comparison of our results for $D_{L L}^{\text {sim }}$ with quasilinear theory would amount to a comparison of $T D_{L L}^{\text {sim }}$ with the time integral of $\left(L^{6} R_{E} / 4 \mu_{E}^{2}\right) \hat{E}\left(\Omega_{3} / 2 \pi\right)$, which we identify via (14) with $T D_{L L}^{q L}$. In other words, our division by $T$ in (14) and (A2) amounts to a convention that facilitates comparisons with data which are best interpreted in terms of a time-dependent radial-diffusion coefficient [cf. Lanzerotti et al., 1971; 1978; Lyons and Schulz, 1989], whereas (A2) formally enables $D_{L L}^{q l}$ to be calculated via (14) as a continuous function of drift frequency. For example, the electric-potential model $\Delta V(t)$ specified by (14) leads to a spectral-density function of the form

$$
\begin{gathered}
\hat{E}(\omega / 2 \pi)=\frac{1}{T}\left|\sum_{i=1}^{N} \int_{t_{i}}^{\infty} \Delta E_{i} \exp \left[\tau^{-1}\left(t_{i}-t\right)+i \omega t\right] d t\right|^{2} \\
=\frac{1}{T} \frac{\tau^{2}}{1+\omega^{2} \tau^{2}} \sum_{i=1}^{N} \sum_{j=1}^{N} \Delta E_{i} \Delta \dot{E}_{j} \cos \left[\omega\left(t_{j}-t_{i}\right)\right], \quad \text { (A3) }
\end{gathered}
$$

where the summations extend over all $N$ impulses. The application of (A3) to the model storm illustrated in Figure $2 b$ leads to the spectral-density function shown in Figure 6 and to the radial-diffusion coefficient plotted in Figure 7 as a continuous function of $\mu$.

Acknowledgments. The authors would like to thank J. B. Blake and W. A. Kolasinski for providing helpful references concerning the "dead time" distortion of a Poisson distribution. Their experience with nuclear instruments and methods has been quite relevant to our modeling of magnetic storms as almost-random sequences of substorms. We would like to thank L. J. Lanzerotti for an interesting discussion of helpful references illustrating the lack of smoothness typically encountered in spectral-density functions computed from observational data. We would also like to thank C. W. Horton, Jr. for suggesting to test nonlinearities by reducing the amplitude of the electric field impulses in our model storm. Our work is supported by the NASA Space Physics Theory Program under grant NAGW-2126. One of the authors, Margaret W. Chen, was a National Research Council (NRC) Research Associate during her work on this paper. Computing resources for this study were provided by the San Diego Supercomputer Center and by the NASA Center for Computational Sciences.

The Editor thanks the two referees for their assistance in evaluating this paper.

\section{REFERENCES}

Akasofu, S.-I., The main phase of magnetic storms and the ring current, Space Sci. Rev., 2, 91-135, 1963.

Blackman, R. B., and J. W. Tukey, The Measurement of Power Spectra, Dover, Mineola, N. Y., 1958. 
Bostrom, C. O., D. S. Beall, and J. C. Armstrong, Time history of the inner radiation zone, October 1963 to December 1968, J. Geophys. Res., 75, 1246-1256, 1970.

Brice, N. M., Bulk motion of the magnetosphere, J. Geophys. Res., 72, 5193-5211, 1967.

Chen, A. J., Penetration of low-energy protons deep into the magnetosphere, J. Geophys. Res., 75, 2458-2467, 1970.

Chen, M. W., M. Schulz, L. R. Lyons, and D. J. Gorney, Ion radial diffusion in an electrostatic impulse model for stormtime ring current formation, Geophys. Res. Lett., 19, 621-624, 1992.

Cornwall, J. M., Diffusion processes influenced by conjugate-point wave phenomena, Radio Sci., 3, 740-744, 1968.

Craven, J. D., Temporal variations of electron intensities at low altitudes in the outer radiation zone as observed with satellite Injun 3, J. Geophys. Res., 71, 5643-5663, 1966.

DeForest, S. E., and C. E. McIlwain, Plasma clouds in the magnetosphere, J. Geophys. Res., 76, 3587-3611, 1971.

Dessler, A. J., and E. N. Parker, Hydromagnetic theory of geomagnetic storms, J. Geophys. Res., 64, 2239-2252, 1959.

Dungey, J. W., Interplanetary magnetic field and the auroral zones, Phys. Rev. Lett., 6, 47-78, 1961.

Dungey, J. W., Effects of electromagnetic perturbations on particles trapped in the radiation belts, Space Sci. Rev., 4, 199-222, 1965.

Ejiri, M., Trajectory traces of charged particles in the magnetosphere, J. Geophys. Res., 83, 4798-4810, 1978.

Evans, R. D., The Atomic Nucleus, McGraw-Hill, New York, 1955.

Fälthammar, C.-G., Effects of time-dependent electric fields on geomagnetically trapped radiation, J. Geophys. Res., 70, 25032516,1965 .

Frank, L. A., On the extraterrestrial ring current during geomagnetic storms, J. Geophys. Res., 72, 3753-3768, 1967.

Frank, L. A., and H. D. Owens, Omnidirectional intensity contours of low-energy protons $(0.5 \lesssim E<50 \mathrm{keV})$ in the Earth's outer radiation zone at the magnetic equator, J. Geophys. Res., 75 , 1269-1278, 1970.

Goldstein, H., Classical Mechanics, Addison-Wesley, Massachusetts, 1980.

Hamilton, D. C., G. Gloeckler, F. M. Ipavich, W. Stüdemann, B. Wilken, and G. Kremser, Ring current development during the great geomagnetic storm of February 1986, J. Geophys. Res., 93, 14,343-14,355, 1988.

Hill, T. W., and M. E. Rassbach, Interplanetary magnetic field direction and the configuration of the dayside magnetosphere, J. Geophys. Res., 80, 1-6, 1975.

Hogg, R. V., and A. T. Craig, Introduction to Mathematical Statistics, Macmillan, New York, 1959.

Kavanagh, L. D., J. W. Freeman, Jr., and A. J. Chen, Plasma flow in the magnetosphere, J. Geophys. Res., 73, 5511-5519, 1968.

Kistler, L. M., F. M. Ipavich, D. C. Hamilton, G. Gloeckler, B. Wilken, G. Kremser, and W. Stüdemann, Energy spectra of the major ion species in the ring current during geomagnetic storms, J. Geophys. Res., 94, 3579-3599, 1989.

Lanzerotti, L. J., C. G. Maclennan, and M. Schulz, Reply (to comments of M. Walt and L. L. Newkirk), J. Geophys. Res., $76,5371-5373,1971$.

Lanzerotti, L. J., D. C. Webb, and C. W. Arthur, Geomagnetic field fuctuations at synchronous orbit, 2, Radial diffusion, $J$ Geophys. Res., 83, 3866-3870, 1978.

Lanzerotti, L. J., and A. Wolfe, Particle diffusion in the geomagnetosphere: Comparison of estimates from measurements of magnetic and electric field fluctuations, J. Geophys. Res., 85, 2346-2348, 1980.

Lyons, L. R., and M. Schulz, Access of energetic particles to storm time ring current through enhanced radial "diffusion", J. Geophys. Res., 94, 5491-5496, 1989.

Lyons, L. R., and D. J. Williams, The storm and poststorm evolution of energetic (35-560 keV) radiation belt electrons, J. Geophys. Res., 80, 3985-3994, 1975.

Lyons, L. R., and D. J. Williams, Storm-associated variations of equatorially mirroring ring current protons, $1-800 \mathrm{keV}$, at constant first adiabatic invariant, $J$. Geophys. Res., 81, 216-220, 1976.

Lyons, L. R., and D. J. Williams, A source for geomagnetic storm main phase ring current, J. Geophys. Res., 85, 523-530, 1980.
Lui, A. T. Y., R. W. McEntire, and S. M. Krimigis, Evolution of the ring current during two geomagnetic storms, J. Geophys. Res., 92, 7459-7470, 1987.

Mayaud, P. N. Derivation, Meaning, and Use of Geomagnetic Indices, Geophys. Monogr. Ser., vol. 22, AGU, Washington, D. C., 1980.

Müller, J. W., Dead-time problems, Nucl. Instrum. Methods, $112,47-57,1973$

Müller, J. W., Some formulae for a dead-time-distorted Poisson process, Nucl. Instrum. Methods, 117, 401-404, 1974.

Nishida, A., Formation of a plasmapause, or magnetospheric plasma knee by combined action of magnetospheric convection and plasma escape from the tail, J. Geophys. Res., 71, 5669-5679, 1966.

Pfitzer, K., S. Kane, and J. R. Winckler, The spectra and intensity of electrons in the radiation belts, Space Res., 6, 702-713, 1966.

Press, W. H., B. P. Flannery, S. A. Teukolsky, and W. T. Vettering, Numerical Recipes, Cambridge University Press, Cambridge, Mass., 1986.

Riley, P., and R. A. Wolf, Comparison of diffusion and particle drift descriptions of radial transport in the Earth's inner magnetosphere, J. Geophys. Res., 97, 16,865-16,876, 1992.

Roederer, J. G., Dynamics of Geomagnetically Trapped Radiation, Springer-Verlag, New York, 1970.

Roederer, J. G., and E. W. Hones, Jr., Motion of magnetospheric particle clouds in a time-dependent electric field model, $J$. Geophys. Res., 79, 1432-1438, 1974.

Schulz, M., Effect of drift-resonance broadening on radial diffusion in the magnetosphere, Astrophys. Space Sci., 36, 455-458, 1975.

Schulz, M., The magnetosphere, in Geomagnetism, edited by J. A. Jacobs, pp. 87-293, Academic, San Diego, Calif.,1991.

Schulz, M., and L. J. Lanzerotti, Particle Diffusion in the Radiation Belts, Springer-Verlag, New York, 1974.

Sckopke, N., A general relation between the energy of trapped particles and the disturbance field near the Earth, J. Geophys. Res., 71, 3125-3130, 1966.

Smith, P. H., N. K. Bewtra, and R. A. Hoffman, Motions of charged particles in the magnetosphere under the influence of a time-varying large scale convection electric tield, in Quantitative Modeling of Magnetospheric Processes, Geophys. Monogr. Ser., vol. 21, edited by W. P. Olson, pp. 513-535, AGU, Washington, D. C., 1979.

Smith, P. H., and R. A. Hoffman, Ring current particle distributions during the magnetic storms of December 16-18, 1971, J. Geophys. Res., 78, 4731-4737, 1973.

Söraas, F., and L. R. Davis, Temporal variations of the $100 \mathrm{keV}$ to $1700 \mathrm{keV}$ trapped protons observed on satelite Explorer 26 during first half of 1965, NASA/GSFC Rep. X-612-68-328, Goddard Space Flight Cent., Greenbelt, Md., 1968.

Stern, D. P., A study of the electric field in an open magnetospheric model, J. Geophys. Res., 78, 7292-7305, 1973.

Takahashi, S., T. Iyemori, and M. Takedo, A simulation of the storm-time ring current, Planet. Space Sci., 38, 1133-1141, 1990.

Tsyganenko, N. A., Global quantitative models of the geomagnetic field in the cislunar magnetosphere for different disturbance levels, Planet. Space Sci., 35, 1347-1358, 1987.

Volland, H., A semiempirical model of large-scale magnetospheric electric fields, J. Geophys. Res., 78, 171-180, 1973.

Williams, D. J., Ring current compositions and sources, in $D y$ namics of the Magnetosphere, edited by S.-I. Akasofu, pp. 407424, D. Reidel, Norwell, Mass., 1980.

Williams, D. J., Phase space variations of near equatorially mirroring ring current ions, J. Geophys. Res., 86, 189-194, 1981.

Williams, D. J., and L. R. Lyons, The proton ring current and its interaction with the plasmapause: Storm recovery phase, $J$. Geophys. Res., 79, 4195-4207, 1974.

Williams, D. J., and A. M. Smith, Daytime trapped electron intensities at high latitudes at 1100 kilometers, J. Geophys. Res., 70, 541-566, 1965 .

M. W. Chen, D. J. Gorney, L. R. Lyons, and M. Schulz, Space and Environment Technology Center, The Aerospace Corporation, P.O. 92957, Mail Station M2-260, Los Angeles, CA 900092957.

(Received December 5, 1991; revised August 20, 1992; 








